\title{
A tale of two drug targets: the evolutionary history of BACE1 and BACE2
}

\author{
Christopher Southan ${ }^{1}$ and John M. Hancock ${ }^{2 *}$ \\ 1 IUPHAR Database and Guide to Pharmacology Web Portal Group, University/BHF Centre for Cardiovascular Science, Queen's Medical Research Institute, \\ University of Edinburgh, Edinburgh, UK \\ 2 Department of Physiology, Development and Neuroscience, University of Cambridge, Cambridge, UK
}

Edited by:

Christian M. Zmasek, Washington

University, USA

Reviewed by:

Victor P. Andreev, University of Miami, USA

Dapeng Wang, Beijing Institute of

Genomics, China

\section{*Correspondence:}

John M. Hancock, Department of

Physiology, Development and

Neuroscience, University of

Cambridge, Downing Street,

Cambridge CB2 3EG, UK

e-mail:jmhancock@gmail.com
The beta amyloid (APP) cleaving enzyme (BACE1) has been a drug target for Alzheimer's Disease (AD) since 1999 with lead inhibitors now entering clinical trials. In 2011, the paralog, BACE2, became a new target for type II diabetes (T2DM) having been identified as a TMEM27 secretase regulating pancreatic $\beta$ cell function. However, the normal roles of both enzymes are unclear. This study outlines their evolutionary history and new opportunities for functional genomics. We identified 30 homologs (UrBACEs) in basal phyla including Placozoans, Cnidarians, Choanoflagellates, Porifera, Echinoderms, Annelids, Mollusks and Ascidians (but not Ecdysozoans). UrBACEs are predominantly single copy, show $35-45 \%$ protein sequence identity with mammalian BACE1, are $\sim 100$ residues longer than cathepsin paralogs with an aspartyl protease domain flanked by a signal peptide and a C-terminal transmembrane domain. While multiple paralogs in Trichoplax and Monosiga pre-date the nervous system, duplication of the UrBACE in fish gave rise to BACE1 and BACE2 in the vertebrate lineage. The latter evolved more rapidly as the former maintained the emergent neuronal role. In mammals, Ka/Ks for BACE2 is higher than BACE1 but low ratios for both suggest purifying selection. The $5^{\prime}$ exons show higher $\mathrm{Ka} / \mathrm{Ks}$ than the catalytic section. Model organism genomes show the absence of certain BACE human substrates when the UrBACE is present. Experiments could thus reveal undiscovered substrates and roles. The human protease double-target status means that evolutionary trajectories and functional shifts associated with different substrates will have implications for the development of clinical candidates for both $A D$ and T2DM. A rational basis for inhibition specificity ratios and assessing target-related side effects will be facilitated by a more complete picture of BACE1 and BACE2 functions informed by their evolutionary context.

Keywords: BACE1, BACE2, Alzheimer's Disease, type II diabetes, protein family evolution

\section{INTRODUCTION}

The amino acid aggregates of $\mathrm{A} \beta$ peptides forming the major component of plaques characteristic of Alzheimer's Disease (AD) result from $\mathrm{N}$-terminal cleavage of the amyloid precursor protein (APP) (Goedert and Spillantini, 2006) mediated by an aspartyl protease referred to as Beta-site APP Cleaving Enzyme 1 (BACE1) (Hussain et al., 1999; Sinha et al., 1999; Vassar et al., 1999; Yan et al., 1999). BACE1 cleaves APP between residues 671 and 672 leading to extracellular release of beta-cleaved soluble APP. The cell-associated carboxy-terminal fragment of APP is subsequently released by the gamma-secretase complex of proteins facilitating intra-membrane proteolysis by the presenilin proteins, PSEN1 and PSEN2 (Selkoe and Wolfe, 2007). Because neurotoxic A $\beta$ peptide production needs the combination of BACE1 and gamma secretase, both have been intensively pursued as $\mathrm{AD}$ drug targets for well over a decade (Durham and Shepherd, 2006; Olson and Albright, 2008; Karran et al., 2011). For gamma secretase inhibitors, recent clinical trial results have been disappointing, although second-generation, Notch-sparing compounds may still hold promise (Xia et al., 2012).
Pursued by many of the same companies in parallel to gamma secretase, global efforts toward BACE1 inhibition have been intense (Probst and Xu, 2012), culminating in the progression of several optimized leads toward their clinical phases (Stamford et al., 2012; Weiss et al., 2012; Hilpert et al., 2013). The prospects for success compared to gamma secretase seem more hopeful for three reasons. Firstly, the mouse BACE1 knock-out remains the only one of nearly 2000 tested viable gene ablations that robustly alter brain $A \beta$ levels (Toyn et al., 2010). Secondly, LY2811376 (PubChem CID 44251605) has provided a proof-ofconcept $A \beta$ reduction in human clinical studies, despite progression being halted because of probable compound-specific (rather than target-specific) retinal toxicology (May et al., 2011). Thirdly, there has been new, indirect genetic target validation in the form of an A673T mutation in APP that was AD-protective due to reduced BACE1 cleavage (Jonsson et al., 2012).

Within a year of the discovery of BACE1 its paralog BACE2 (Q9Y5Z0) was also published by multiple groups (Acquati et al., 2000; Farzan et al., 2000; Hussain et al., 2000). This has 50\% identity to BACE1 over 518 residues in humans. The next-highest 
alignment score against human proteins is cathepsin E (CATE, P14091) with 27\% identity over 396 residues. A phylogenetic analysis has established that the BACEs are parologous to pepsins and cathepsins, although they may have shared origins with homologs found in the marine proteobacterian genus Shewanella (Rawlings and Bateman, 2009). The bi-lobed tertiary structure of the A1 proteases suggests this family has arisen from a duplication and fusion event. Even though the two symmetrical lobes of the PDB structures have recognizably similar folds, the residual internal sequence similarity from the ancient duplication is restricted to the short motif around each active site Asp.

Since investigations on the normal roles of BACE1 and BACE2 are too extensive to review here (particularly for BACE1) we present just a selection of citations to give an overview (Table 1).

The current picture of BACE function is increasingly complex but trends can be discerned. The first trend is that the continued confirmation of new in vivo substrates points toward pleiotropic roles. The second is that, while the picture of neuronal substrate processing for BACE1 and pancreatic substrates for BACE2 holds true, there is increasing evidence of overlap. In particular, BACE1 functions may extend to non-CNS tissues and cell types in which the same and/or different substrates can be processed. The third trend is the emergence of role differences between humans, mice and fish.

A notable 2011 report unexpectedly promoted BACE2 to an equivalent drug target status to that which BACE1 had immediately acquired in 1999. Since TMM27 (Q9HBJ8) was shown to be a regulator of normal beta cell function the research team went on to show that insulin-resistant mice treated with a BACE2 inhibitor (CID 50938551) displayed both augmented $\beta$ cell mass and improved control of glucose homeostasis due to increased insulin levels (Esterhazy et al., 2011). These findings therefore constituted an initial drug target validation of BACE2 inhibition for type II diabetes (T2DM). While the molecular mechanisms by which BACE2 deficiency or inhibition affect $\beta$ cell function and proliferation are unknown, they may involve not only the stabilization of TMEM27 but additional BACE2 substrates (Stützer et al., 2013).

The double drug target status of the BACEs, together with the still-incomplete functional pictures of both enzymes, presents an opportunity for a phylogenomic investigation. This is facilitated by the increasing breadth (i.e., more species) and depth (i.e., more phyla) of completed genomes, draft assemblies and transcript data. For BACE1, the existence of sequence similarity matches in Ciona intestinalis and Strongylocentrotus purpuratus had already been noted (Stockley and O'Neill, 2008; Venugopal et al., 2008). Here we focus on the discovery of novel homologs from basal phyla. From finding a predominantly single-copy UrBACE in most eumetazoans we identify a major duplication event approximately corresponding to the origin of the jawed vertebrates (Gnathostomata). We also suggest that frequent duplication and loss events may have contributed to the evolution of this gene family.

\section{METHODS}

\section{REFERENCE SEQUENCES AND TERMINOLOGY}

Detailed information on the search sequences can be found in the appropriate UniprotKB/Swiss-Prot records for BACE1_HUMAN
Table 1 | Summaries of findings related to normal functions of BACE1 (Upper section) and BACE2 (lower section) in chronological order (KO = gene knock-out).

\begin{tabular}{ll}
\hline Observation & References \\
\hline BACE1 & \\
$\begin{array}{ll}\text { KO-mice showed subtle neurochemical deficits and } \\
\text { behavioral changes }\end{array}$ & $\begin{array}{l}\text { Dominguez et al., } \\
2005\end{array}$ \\
\hline $\begin{array}{l}\text { Cleaved APP ectodomain involvement in normal } \\
\text { nerve cells and A } \beta \text { peptides dampening neuronal } \\
\text { hyperactivity }\end{array}$ & Ma et al., 2007 \\
\hline
\end{tabular}

Sixty-eight epithelial cell line substrates detected, Hemming et al., many membrane-anchored and involved in 2009 contact-dependent intercellular communication

Voltage-gated sodium channel subunits (SCN4B, Kovacs et al., 2010 060939 and related subunits) substrates for

regulation of Nav1 channel metabolism

Neuregulin, NRG1, 0022979, substrate for control Fleck et al., 2012

of nerve cell myelination

Amyloid-like protein 2 (APP2, Q06481) substrate Hogl et al., 2011

for ectodomain fragments

Brain substrates in inhibitor-treated and KO mice Kuhn et al., 2012

involved in neurites and synapses

Thirteen non-amyloidogenic substrates reviewed Dislich and

Lichtenthaler, 2012

Pancreatic ectodomain shedding regulates broad

Stützer et al., 2013

set of $\beta$-cell-enriched substrates

Zebrafish $\mathrm{KO}$ indicates substrates related to

neurite outgrowth and axon guidance, including

Hogl et al., 2013

plexin A3, B0S5N4, and glypican-1 (F1OCC6)

Zebrafish $\mathrm{KO}$ shows peripheral hypomyelination

Van Bebber et al.

2013

BACE2

Processes APP at the beta-secretase site

Hussain et al., 2000

Tissue distribution implies functions distinct from Sun et al., 2005 neuronal BACE1

KO mice normal but neonatal mortality increase in Dominguez et al.,

BACE1/2 double-KO 2005

Processes APP but reduces A $\beta$ production Sun et al., 2006

Secretase of the plasma membrane protein Esterhazy et al.,

TMM27 (Q9HBJ8) in mice and in human $\beta$ cells 2011

Pancreatic ectodomain shedding regulates narrow Stützer et al., 2013 set of $\beta$-cell-enriched substrates, including SEZ6L

(Q9BYH1) and SEZ6L2 (Q6UXD5)

Role in processing mouse pigment cell-specific Rochin et al., 2013

Melanocyte Protein, PMEL, 060696

Zebrafish $\mathrm{KO}$ melanocyte migration phenotype.

Double KO (Bace1 ${ }^{-/-}$; Bace $2^{-/-}$) viable and does

Van Bebber et al.,

not enhance the single mutant phenotypes,

indicating non-redundant functions in fish 
(P56817) and BACE2_HUMAN (Q9Y5Z0). Additional information is available in the MEROPS peptidase database via the identifiers A01.004 (http://merops.sanger.ac.uk/cgi-bin/pepsum? mid=a01.004) and A01.041 (http://merops.sanger.ac.uk/cgi-bin/ pepsum?mid=A01.041), respectively (Rawlings et al., 2008). Comparative genomic data can be accessed via the Ensembl entry points for ENSG00000186318 (http://www.ensembl.org/ Homo_sapiens/Gene/Summary?g=ENSG00000186318;r=11:117 156402-117186975) and ENSG00000182240 for BACE1 and BACE2, respectively (http://www.ensembl.org/Homo_sapiens/ Gene/Summary?db=core;g=ENSG00000182240;r=21:4253972842648524). In summary BACE1 is transcribed from 9 exons on human chromosome 11q23.3 and BACE2 from 9 exons on 21q22.3.

To reduce repetition we use the following terminology. While BACE is technically a BACE1 synonym (whose usage preceded the latter) we use the term $\mathrm{BACE}(\mathrm{s})$ to refer to the pan-vertebrate parologous pairs of BACE1 and BACE2. The term BACE-like is reserved for high-scoring similarity matches that we detected but that were not unequivocally assignable to either. Where our analysis has clearly resolved these to single (or low multiple) ORFs in basal phyla we use the term UrBACE (see Results).

\section{SEOUENCE SEARCHING AND CHECKING}

The utility of selected resources for the phylogenetic investigation of proteases and their substrates has been previously noted (Southan, 2007). We made use of Ensembl GeneTree as an automated starting point from which we manually checked selected ORFs and proceeded to search for new homologs in the sequence databases (Ruan et al., 2008). For gene predictions or transcript translations we inspected NCBI BLASTP output for similarity matches, truncations, insertions or deletions, using default search parameters. All BACE-like, genomic pipeline-predicted ORFs were checked by BLASTX searches against transcript collections. In a few cases GENESCAN runs on genomic DNA for de novo protein prediction extended the ORFs.

InterProScan was used to detect extended family matches, local domain matches, N-terminal patterns indicative of signal peptides, C-terminal transmembrane domains and to detect breaks in the global alignment profiles. Initially we were confounded by cases where direct THMM transmembrane and SignalP prediction indicated terminal TMs apparently missed by InterProScan. We eventually discovered that a cryptic licensing restriction in the online version of InterProScan meant sequences had to be changed by a single residue from identical UniProt entries before TMs were generated.

By performing TBLASTN searches of ORFs against both Expressed Sequence Tags (dbEST) and the Transcriptome Shotgun Assembly sequence division (TSA) we found many new BACE-like sequences. These included complete and partial cDNAs from basal phyla that do not yet have complete genome coverage. Such searches were also used to extend truncated genomic-predicted ORFs. The expanding coverage of mammals and major vertebrate phyla by Ensembl has produced dense coverage for BACEs via the automated population of GeneTree. We therefore focused our collation efforts on expanding the more sparsely populated deep phylogeny which is currently restricted to just Ciona in Ensembl.

We selected a limited number of close, cathepsin-like paralogs to these BACE-like sequences as out-group sequences to resolve the possible evolutionary patterns. These combined approaches allowed us to generate a large set of sequences most of which were novel. We also used representative human substrates of BACE1 and BACE2 to search against sets of predicted proteins from model organism genomes to identify their phylogenetic distributions. We included presenilin 1 (PSEN1), which is also associated with secretase action, via APP being a substrate. Since this is highly conserved with a deep phylogenetic pattern it served as a useful similarity score calibration.

\section{ALIGNMENT AND PHYLOGENETIC ANALYSIS}

The initial stages used COBALT for checking as the sequences were being iteratively assembled and cross-checked (Papadopoulos and Agarwala, 2007). The final alignments were made using PRANK (Löytynoja and Goldman, 2010). Following recommendations of a recent evaluation (Gonnet, 2012), phylogenetic analysis was carried out by applying BioNJ (Gascuel, 1997) (http://www.phylogeny.fr/version2cgi/onetask.cgi?task type=bionj) to PRANK protein alignments. Tree reconstructions were carried out using default parameters.

\section{SEOUENCE BLOCKS AND GAPPED ALIGNMENTS}

As an adjunct to phylogenetic relationships, multiple alignments can also be used to extract conserved sequence blocks. These were generated with Blockmaker and then analyzed by WebLogo to provide a comparative visual description of residue conservation at each position (Henikoff et al., 1995; Crooks et al., 2004). It should be noted that this approach is selective, compared to tree construction, in that gapped or very divergent sequences are excluded. Block sequence sections and individual residues from the logos were then mapped to a BACE1 2D transformation available in PDBSum (Laskowski, 2009). The important C-terminal domains of BACE-like sequences proved difficult to discern in global multiple alignments or block-type approaches. We addressed this by using the T-Coffee algorithm because this is optimized for gapping (Rausch et al., 2008).

\section{Ka/Ks ANALYSIS}

$\mathrm{Ka} / \mathrm{Ks}$ ratios were estimated on subsets of well-founded cDNAs of BACE1 and BACE2 separately. The cDNA sequences were derived only from mammals to minimise effects of multiple mutations (although these cannot be eliminated completely) and included only coding sequence. $\mathrm{Ka} / \mathrm{Ks}$ calculations were carried out using SLAC (Kosakovsky Pond and Frost, 2005) for complete coding regions and for sub-regions corresponding to functional domains and to individual exons. Boundaries of functional domains and intron/exon boundaries were taken from the CCDS database (Pruitt et al., 2009)

\section{DATA AVAILABILITY, RE-USE, UPDATING, AND CONNECTIVITY}

We have taken four complementary approaches to data sharing that we hope will ensure persistence, re-use, updating, and facilitate connectivity (Leebens-Mack et al., 2006; Stoltzfus et al., 
2012; Drew, 2013). Firstly, we have used the supplementary data option provided by this journal, with the specific data being referred to in results. Secondly, we have made a deposition in TreeBase (Anwar and Hunt, 2009) of a Nexus file representing the alignment used for the phylogeny analysis. The permanent URL for this Nexus file is http://purl.org/phylo/treebase/phylows/ study/TB2:S14732. As a third measure we have deposited the supplementary data on figshare as an open archive (Singh, 2011), at http://figshare.com/articles/Supplementary_Data_for_Southan_ Hancock_BACE_evolution_paper/855620.

It is important to note that the majority of the sequences we have collated are not yet available as stable protein accession numbers for full-length ORFs and their primary nucleotide records are spread across many database divisions. In addition, the rate of generation and revision (e.g., new cDNA and genome assembly updates) is such that our TreeBase deposition will become outdated. We have thus included (both in the supplementary data and the figshare link) a complete FASTA compilation of the sequences. These can either be used as-is for different alignment approaches or updated via new database searches.

Fourthly, we have submitted a representative complete cDNA and protein sequence of the Ciona intestinalis UrBACE to the Third Party Annotation (TPA) division of the European Nucleotide Archive (ENA). This was assembled using EST data to consolidate the gene prediction. This will have the accession number HE967761. We will ensure this is updated to link to the eventual PubMed ID, giving researchers the possibility of connecting to our work directly via a sequence search.

\section{RESULTS}

\section{CLASSIFYING BACE-LIKE ORFS}

The exercise of finding and checking new BACE-like proteins presented the following technical challenges:

- Discerning what data types for which organism were in which source and/or database division (e.g., cDNA data could be in mRNA(nr), dbEST, TSA, or all three).

- Ascertaining completion status at the genome assembly level and coverage at the transcript level (e.g., we could not easily resolve the Monosiga ovata EST-derived ORFs against the JGI Monosiga brevicolis genome-derived ORFs).

- Encountering the same or different ORFs from the same organism in multiple pipelines (e.g., JGI, BCM, UCSC, XP, Ensembl, RefSeq, and TrEMBL). It was often unclear which organisms were unique to which portals or which database records were transitively circular (i.e., cross-referenced back to the same primary sequence data) or derived via independent pipeline results.

- For both genomic prediction and cDNA data we commonly encountered the error types of terminal truncations, internal exon losses and chimeras.

Fortunately multiple alignments are tolerant of at least moderate gaps or truncations and can still be informative with respect to tree topology and branch lengths reflecting protein sequence similarity scores. Consequently, some partial sequences were used to populate otherwise sparse sections of the tree.
We classified sequences as BACE-like by two approaches. The first was identifying them as probable orthologs extending across major phyla. The second was discriminating them from cathepsins (i.e., as not cathepsin-like). Our triage utilized the following criteria:

1. Reciprocal BLAST similarity (i.e., using the query best match as a new database query). This always grouped BACEs at the top of the hit list.

2. Matches of $\sim 35 \%$ identity or more, extending across the major part of the ORF without over-gapping (e.g., the Planarium sequence, with one of the lowest similarity scores in the set, matches human BACE1 with an E-value of $3 \mathrm{e}-58$ representing $34 \%$ identity over 387 residues with $7 \%$ gaps).

3. The similarity scores to cathepsin homologs in any single species were distinctly lower (e.g., dropping to $\sim 25 \%$ identity over $\sim 350$ residues with $\sim 20 \%$ gapping).

4. Sequences were typically $\sim 100$ residues longer than cathepsin homologs.

5. A unique pattern of a predicted $\mathrm{N}$-terminal signal peptide and a C-terminal transmembrane (CTM) either side of the protease domain was present. The CTM was absent from all analyzed cathepsin homologs while they typically also had $\mathrm{N}$-terminal signal peptides.

6. Extended global alignment matches, together with individual diagnostic sections including at least one of the profiles for BACE (this was nearly always to BACE1 not BACE2).

7. Cathepsin homologs and vertebrate BACE2 sequences consistently showed two matches to the Prosite PS00141 regular expression diagnostic for the aspartyl active site. In contrast, all BACE1 and BACE-like sequences showed only the single proximal N-terminal match.

8. The gene structure of all BACE-like sequences, judged as complete ORFs, consisted of at least 9 exons. Most cathepsin homologs also showed this but some were single-exon.

9. The construction of provisional phylogenetic gene trees and testing different parameterizations, including cathepsins as out-groups, was used to support grouping into BACE-like or BACE sub-families.

Using the methods described we assembled the sequences specified in Table 2.

\section{NEGATIVE AND BORDERLINE RESULTS}

With the caveat of data incompleteness in organisms represented only by draft genomes, BACE-like proteins were not detected (i.e., the BACE1 search probe matched only cathepsins at $\sim 25 \%$ identity) in the folowing: Saccharomyces cerevisiae, Caenorhabditis elegans, Drosophila melanogaster, Daphnia pulex, Arabidopsis thaliana, Amphimedon queenslandica, Dictyostelium discoideum, Plasmodium falciparum, and Strigamia maritima. In addition, by using the taxonomic filters on the UniProt BLAST options, the entire protein collections from bacteria, plants, fungi, archaea and nematodes were also found to be negative. In the light of these results we dispute the published claim of the discovery of a BACElike sequence in Drosophila melanogaster and the consequent annotation as such in the protein databases (Carmine-Simmen 
Table 2 | BACEs, BACE-like sequences and homologs used for phylogenetic analysis.

\begin{tabular}{|c|c|c|c|c|c|}
\hline Short sequence name & Species name & Common name & NCBI Tax ID & UniProt ID & Protein length \\
\hline \multicolumn{6}{|l|}{ Ur-BACE } \\
\hline Mono_ovat_A & Monosiga ovata & Choanoflagellate & 81526 & & 459 \\
\hline Tric_adhe_A & Trichoplax adhaerens & Tricoplax & 10228 & & 545 \\
\hline Tric_adhe_B & Trichoplax adhaerens & Tricoplax & 10228 & B3RU95 & 505 \\
\hline Aipt_pall & Aiptasia pallida & Sea Anemone & 12566 & & 488 \\
\hline Hydr_mag & Hydra magnipapillata & Hydra & 6085 & & 412 \\
\hline Clyti_hem & Clytia hemisphaerica & Sponge & 252671 & & 474 \\
\hline Acro_mill & Acropora millepora & Stony Coral & 45264 & & 365 \\
\hline Clon_sine & Clonorchis sinensis & Oriental Liver Fluke & 79923 & & 506 \\
\hline Mere_mere & Meretrix meretrix & Asiatic Hard Clam & 291251 & & 362 \\
\hline Rudi_phil & Ruditapes philippinarum & Manila Clam & 129788 & & 186 \\
\hline Vill_lien & Villosa lienosa & Freshwater Mussel & 326719 & & 326 \\
\hline Cras_giga & Crassostrea gigas & Pacific oyster & 29159 & & 520 \\
\hline Lott_giga & Lottia gigantea & Owl limpet & 225164 & & 495 \\
\hline Ilya_obso & Ilyanassa obsoleta & Eastern mudsnail & 34582 & & 250 \\
\hline Lymn_stagn & Lymnaea stagnalis & Pond Snail & 6523 & & 544 \\
\hline Eupr_scolo & Euprymna scolopes & Squid & 6613 & & 215 \\
\hline Stron_purp & Strongylocentrotus purpuratus & Sea Urchin & 7668 & & 538 \\
\hline Para_livi & Paracentrotus lividus & Purple Sea Urchin & 7656 & & 297 \\
\hline Hum_BACE1 & Homo sapiens & Human & 9606 & P56817 & 501 \\
\hline Mouse_BACE1 & Mus musculus & Mouse & 10090 & P56818 & 501 \\
\hline Rat_BACE1 & Rattus norvegicus & Rat & 10116 & P56819 & 501 \\
\hline Dog_BACE1 & Canis familiaris & Dog & 9615 & & 501 \\
\hline Bov_BACE1 & Bos taurus & Cow & 9913 & $\mathrm{Q} 2 \mathrm{HJ} 40$ & 501 \\
\hline Mono_dome_BACE1 & Monodelphis domestica & Opossum & 13616 & & 466 \\
\hline Ailu_melan_BACE1 & Ailuropoda melanoleuca & Panda & 9646 & & 501 \\
\hline Orin_anat_BACE1 & Ornithorhynchus anatinus & Platypus & 9258 & & 475 \\
\hline Gall_Gal_BACE1 & Gallus gallus & Chicken & 9031 & & 426 \\
\hline Taen_gutt_BACE1 & Taeniopygia guttata & Zebra Finch & 59729 & & 522 \\
\hline Xeno_trop_BACE1 & Xenopus tropicalis & Western clawed frog & 8364 & Q0P4T5 & 502 \\
\hline Anol_caro_BACE1 & Anolis carolinensis & Lizard & 28377 & & 318 \\
\hline Pelo_sine_BACE1 & Pelodiscus sinensis & Chinese Soft- Shelled Turtle & 13735 & & 484 \\
\hline Chry_pict_BACE1 & Chrysemys picta bellii & Western painted Turtle & 8478 & & 437 \\
\hline Dani_reri_BACE1 & Danio rerio & Zebrafish & 7955 & & 531 \\
\hline Taki_rubr_BACE1 & Takifugu rubripes & Fugu & 31033 & & 443 \\
\hline Tetr_nigr_BACE1 & Tetraodon nigroviridis & Pufferfish & 99883 & Q4RYS5 & 448 \\
\hline Gast_acul_BACE1 & Gasterosteus aculeatus & Sticklback & 69293 & & 490 \\
\hline Oryz_lati_BACE1 & Oryzias latipes & Medaka & 8090 & & 442 \\
\hline
\end{tabular}


Table 2 | Continued

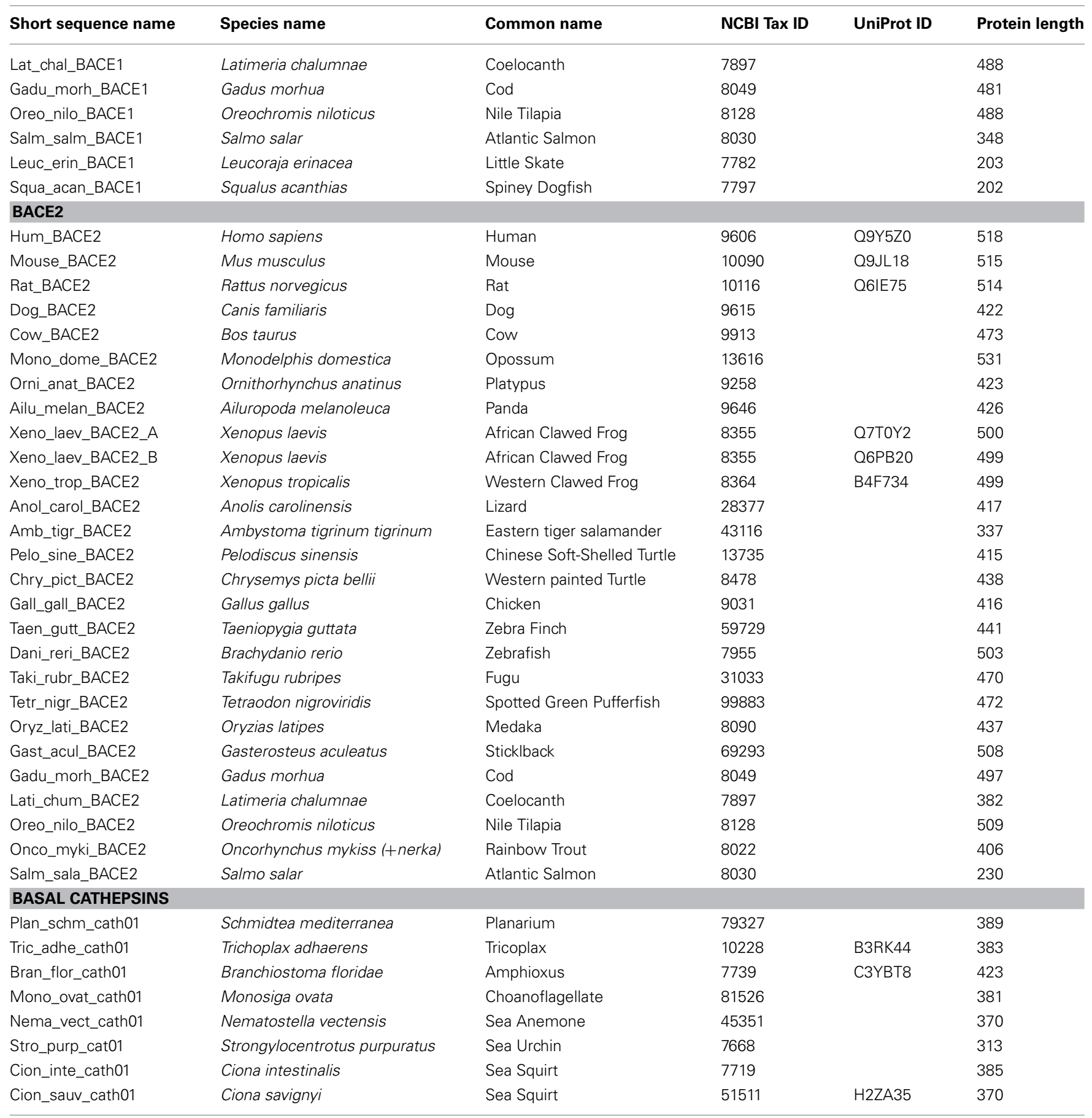

et al., 2009). The sequence in question (Q9VLK3) fails all the criteria we described above and, on the basis of similarity, the most plausible classification is a lysosomal cathepsin. This is regardless of whether the enzyme may exibit the beta-secretase activity of APP-clipping under certain experimental conditions, as has been reported for other cathepsins (Schechter and Ziv, 2011).

The following database matches were recorded as borderline but, because of low identity scores, truncations and absence of corroborative data, they were not included in the multiple alignments. A partial sequence (GAA56694) from the Liver Fluke Clonorchis sinensis gives reciprocal BACE1 scores but has no diagnostic matches in InteProScan and the primary data was not retrievable from the genome portal. The marine metagenome partial ORF EBK78785 also has BACE1 as top reciprocal match and a C-terminal TM domain but it has only $27 \%$ identity with extensive gapping and neighbor matches indicative of it being an algal protein. 


\section{IDENTIFICATION OF SEQUENCE FEATURES}

Figure 1 shows a representative set of InterProScan outputs for two Ur-BACEs, the human BACEs and a cathepsin.

The diagnostic utility of InterProScan results is not only for family and subfamily memberships but also for judging sequences to be full-length. As expected, the pepsin/cathepsin architecture is confirmed by all the global alignment matches. Three of the PRINTS local alignment patterns proved useful to distinguish between BACE family (PRO815), BACE1 (PRO1816), and BACE2 (PRO1817) (Attwood et al., 2012). Notably, the specificity of the matches extends to the Ur-BACEs, even though the profile is historically compiled from mammalian sequences. Ur-BACEs predominantly matched the BACE1 profile and none the BACE2 profile. For sequences with complete N-terminals, InterProScan detected either a tmhmm $(\sim 50 \%)$ or a SignalP $(\sim 30 \%)$ hit, and sometimes both. We interpret this as indicating a secretionassociated signal peptide cleavage as a universal feature. Similarly, all UrBACEs indicated the presence of a C-terminal transmembrane section. We provide a summary of matching across the entire sequence set in Figure 2.

\section{EXTENDED PHYLOGENY}

A gene tree of the entire set of protein sequences from Table 2 is shown in Figure 3A. The UrBACE sequences alone are shown in Figure 3B. The alignment underlying this analysis is available as a Nexus file at http://purl.org/phylo/treebase/phylows/study/TB2: S14732 and both sets of sequences are supplied in FASTA format in the Supplementary Data file.

Figure 3 shows four distinct clusters with the two vertebrate BACEs generally well resolved from the Ur-BACEs and their cathepsin homologs. While we have restricted the vertebrate sequences to major groupings (more orthologs can be accessed via Ensembl GeneTree if required) they recapitulate expected features. For example, BACEs from the new Turtle genomes cluster with birds, as concluded in the recent publication (Shaffer et al., 2013). However, there are departures from a simple evolutionary model based on duplication in the fish linage. In the vertebrate clades, Xenopus tropicalis contains a clearly identifiable BACE1 and BACE2. However, the two homologs identified in X. laevis both clustered with $X$. tropicalis BACE2, share $90 \%$ sequence identity as paralogs, and are therefore designated as BACE2a and BACE2b.

Branch lengths within the BACE2 family appear longer than those for BACE1. To quantify this we compared equivalent sequence distances between the protein sets. The two X. laevis BACE2s were excluded from the analysis, as was the $P$. marinus UrBACE although this clustered with the duplicated BACEs. The average sequence difference for BACE1s was 0.317 whereas that for BACE2s was 0.551. The mean BACE2/BACE1 ratio for all comparisons was 2.36. Of 190 comparisons 150 showed greater distances for BACE2 than BACE1 while 40 showed a greater distance for BACE1 than BACE2. This suggests that BACE2 protein sequences have evolved more rapidly than BACE1. As this result is based on protein sequences, it likely suggests that purifying selection has acted more strongly on BACE1 than BACE2. The full distance matrix for the data set as generated by BIONJ is available in the Supplementary Data file.
The UrBACE sequences lie outside the two well-supported BACE clades with the exception of Petromyzon marinus UrBACE, which clusters close to the BACE1 grouping. Notably, two UrBACE sequences were identified for Monosiga ovata and three for Trichoplax adhaerens. The latter can be localized as an ordered cluster in the genome (Ensembl scaffold_4:758759-790622) but since none are complete ORFs in the browser mark-up (although our version of Tric_adhe_A was extended to 545 residues by $a b$ initio gene prediction) the exon pattern and possible duplication history of the three is unclear. Both of these UrBACE paralogous sets have long branch lengths (e.g., the Monosiga pair shares only $\sim 45 \%$ identity) suggesting that these duplications are ancient events independent of the duplication event giving rise to the BACE1 and BACE2 families. Amongst UrBACEs, most major groupings are supported, although protostomes do not resolve as a monophyletic group. An anomalous result is the high bootstrap value separating the tunicates (Ciona and Halocynthia) not only from other chordates but from all other UrBACEs. This may suggest that these sequences derive from a different duplication lineage to the other UrBACEs and the BACEs.

\section{BLOCKS ANALYSIS}

Figure 4 shows that despite the divergence of their sequences conserved blocks are consistently spaced between the different BACEs. Exceptions are the insert in Schistosoma mansoni and deletion in Trichoplax adhaerens (Tric_adhe_B). The Sequence Logo results are shown in Figure 5 and the mapping of these onto a BACE1 PDB structure in Figure 6.

As expected, the catalytic sites correspond to two of these blocks (blocks 1 and 4). Blocks 2 and 3 align with the ligand contact shell, indicated by the red triangles, while the remaining two blocks are associated with internal fold positions but also include conserved cysteines involved in disulphide bonding. Inspection of the ConSurf option in PDBSum indicates that essentially all 6 UrBACE blocks and the conserved (purple) residues in Figure 5 correspond to buried internal parts of the structure. Note that this analysis provides different but complementary results to the InterProScan and phylogeny results. The former show matches to pre-existing sequences and recognize the terminal domain features that typically do not crystallize. The blocks analysis shows divergence among the UrBACEs despite the common fold architecture. However, despite the nominal presence of catalytic residues, the extensive insertions in the Schistosoma japonicum sequence (if confirmed via cDNA data) suggest it could be catalytically non-functional, which could explain the anomalous branch length in the phylogenetic tree.

Many experiments have indicated the crucial role played by the BACE1 C-terminal, including S-palmitoylation at four Cys residues at the junction of the transmembrane and cytosolic domains. Although this has no discernible influence on BACE1 processing of APP in mouse, it traffics BACE1 dimers into cholesterol-rich lipid rafts (Vetrivel et al., 2009). While the question of the occupancy stoichiometry of the lipid chains between the Cys positions remains, there are no reports that BACE2 has the equivalent post-translational modification (PTM). For the UrBACE sequences block generation breaks down in this part of the multiple alignment although it did indicate all four Cys to be 


\section{A 459 aa}

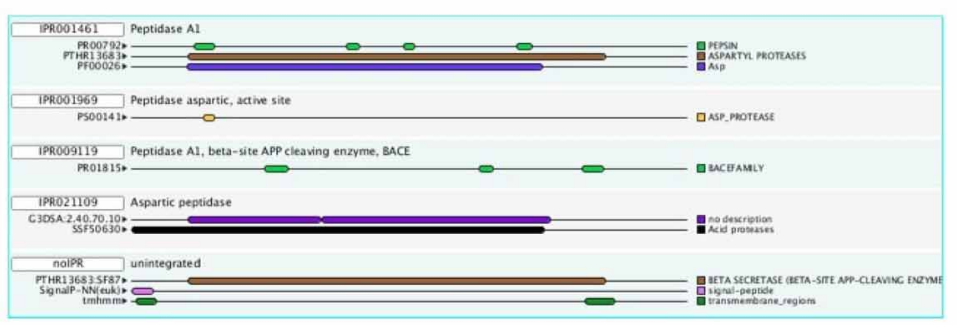

B 538 aa

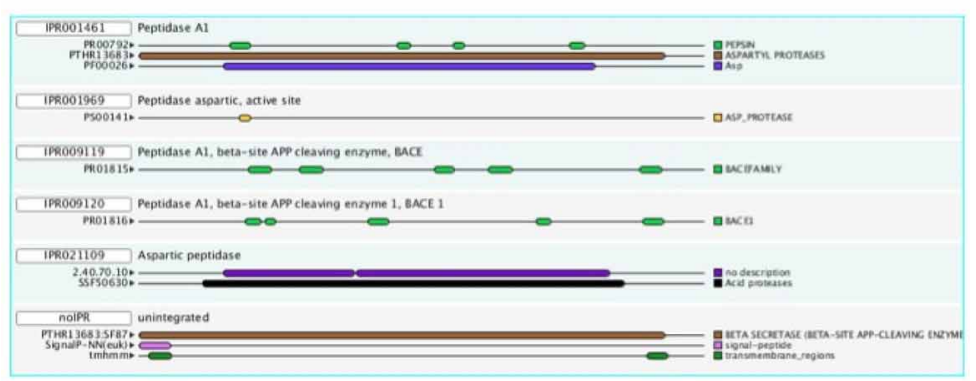

C 501 aa

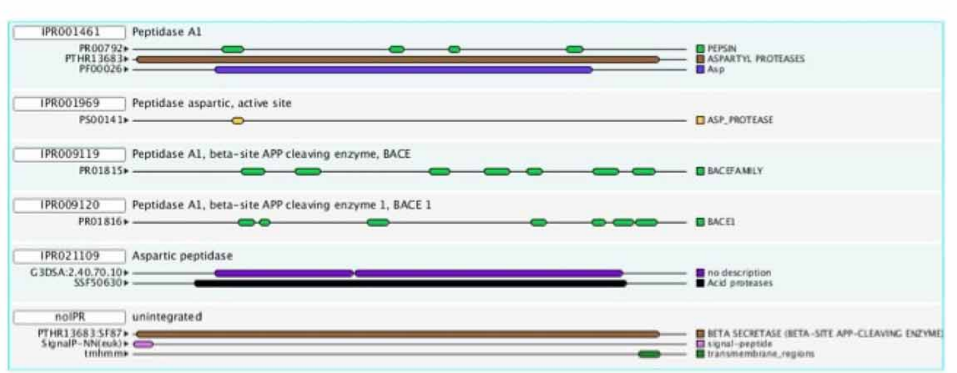

D 518 aa

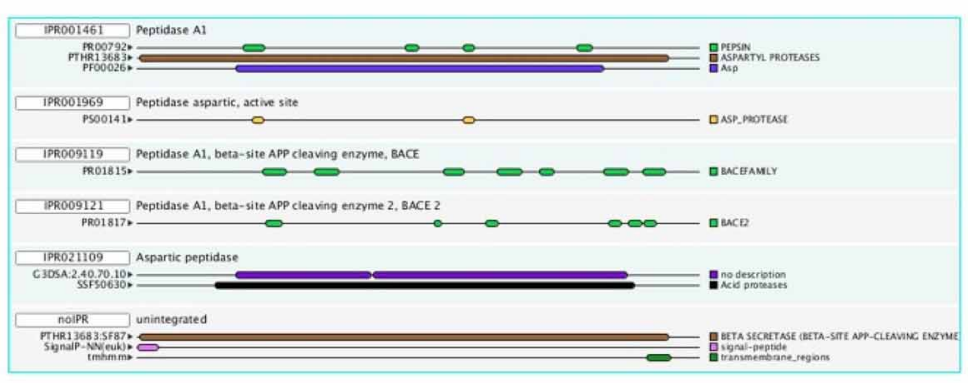

\section{E 381 aa}

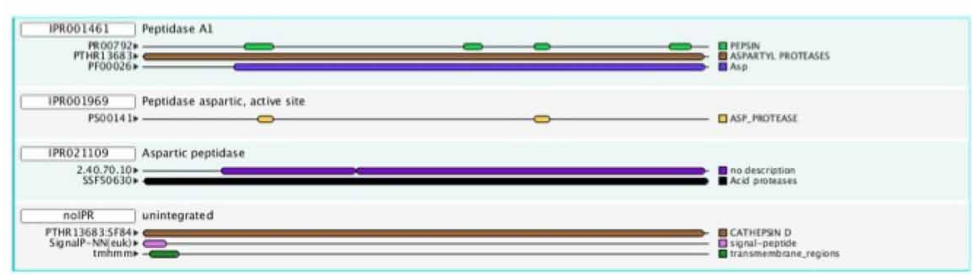

FIGURE 1 | InterProScan Sequence Features. Representative examples are shown for two Ur-BACEs (A) Monosiga ovata A (Mono_ovat_A), (B) Strongylocentrotus purpuratus (Stron_purp), (C) human BACE1
(Hum_BACE1) (D) human BACE2 (Hum_BACE2), and (E) a cathepsin from Monosiga ovata (Mono_ovat_cath01). The protein length is indicated. 


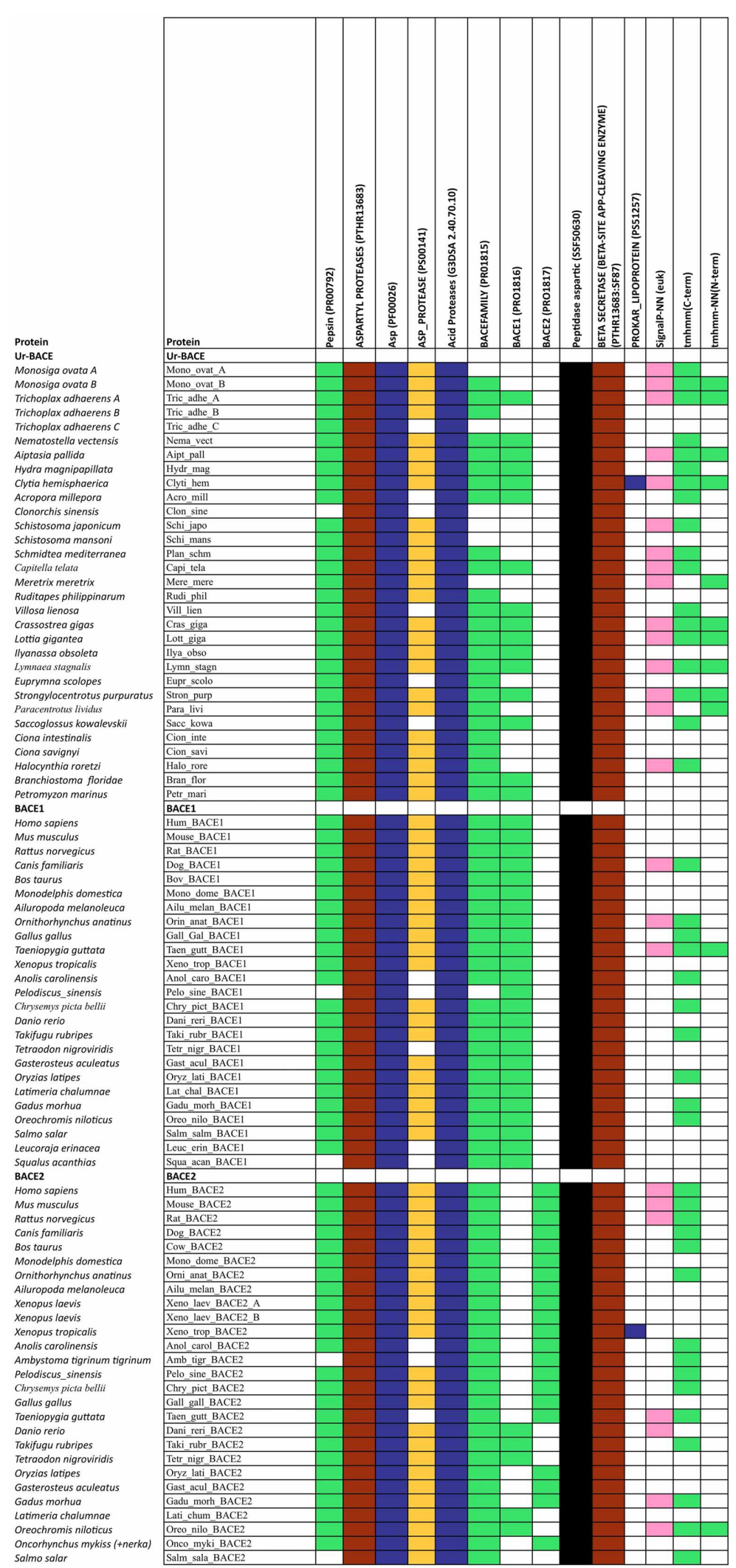

FIGURE 2 | Summary of InterProScan pattern hits for the proteins included in Table 2. 
A

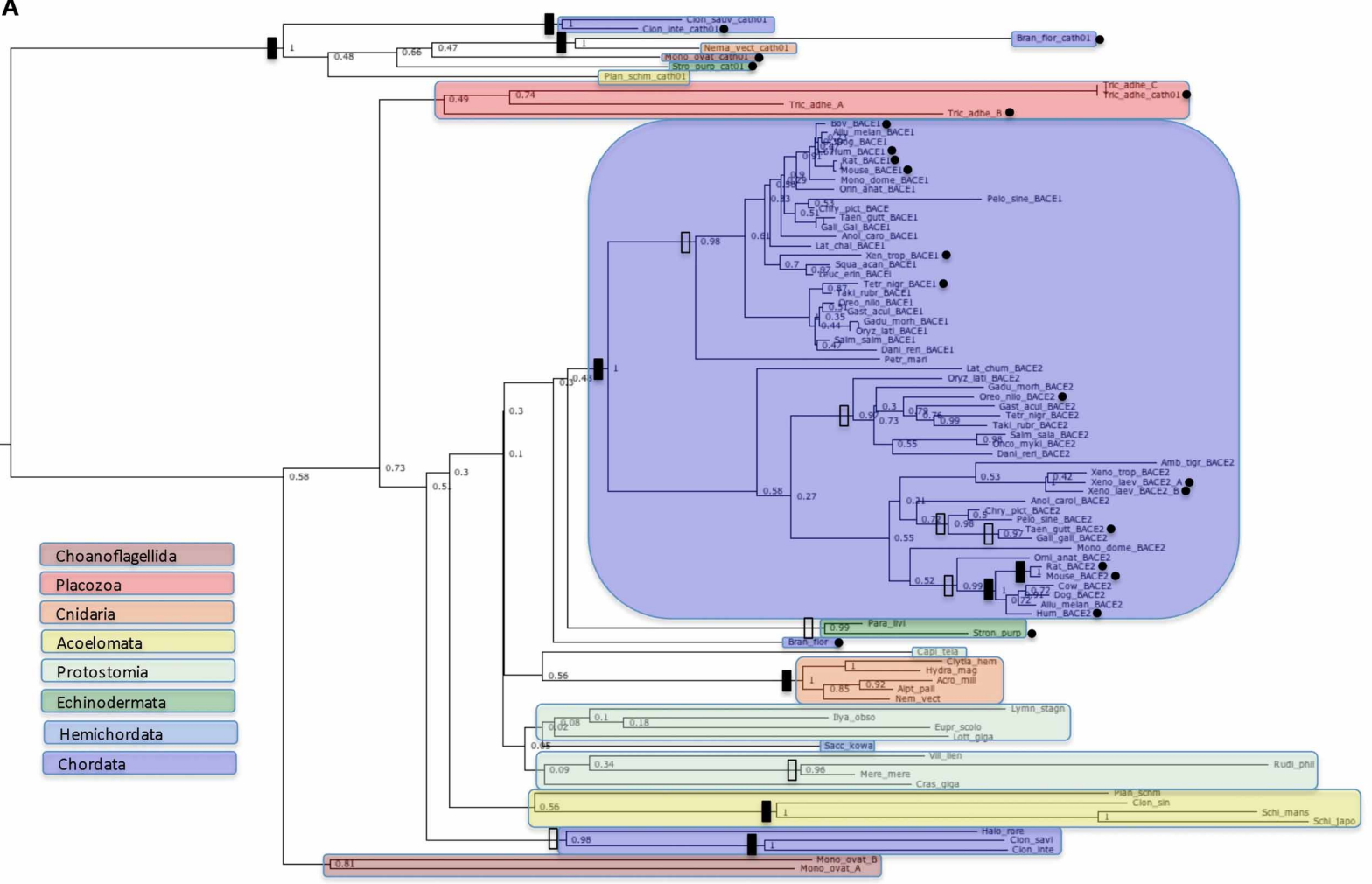

B

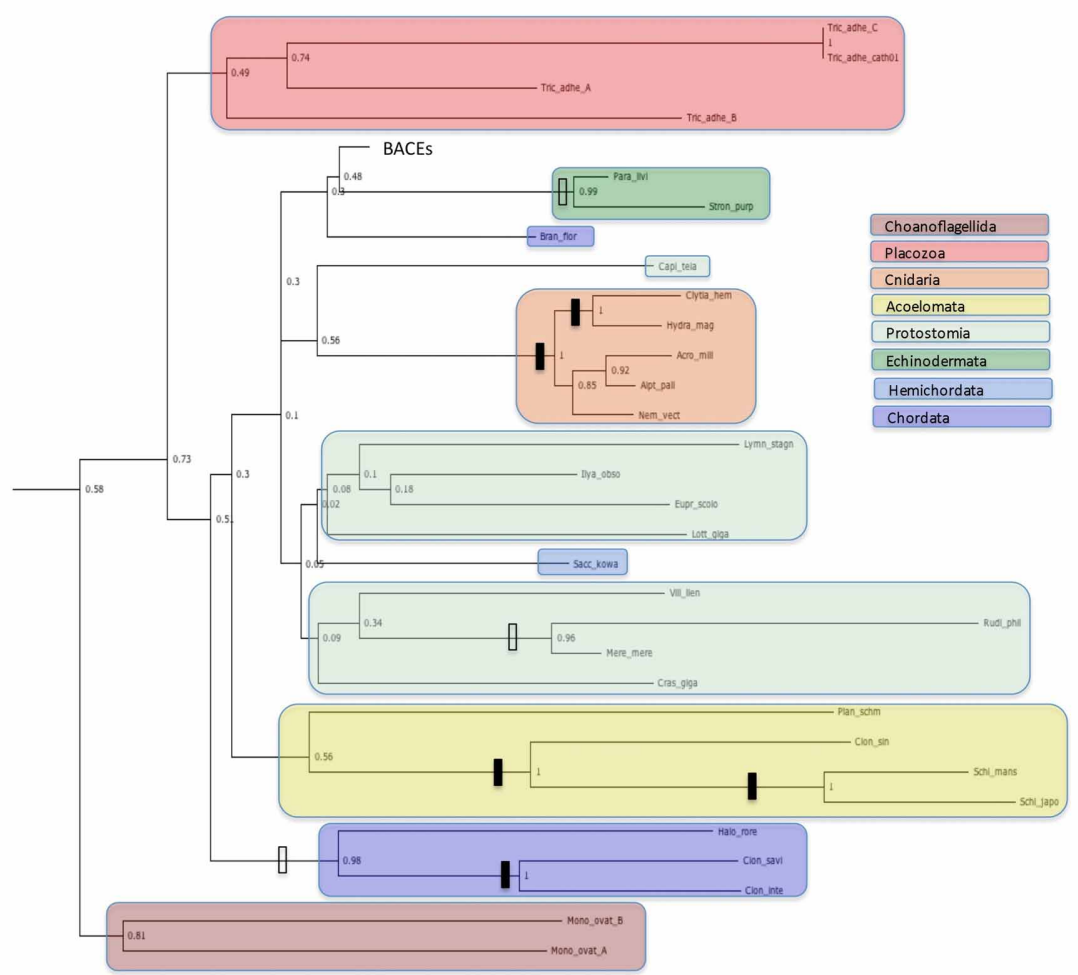

FIGURE 3 | Protein trees of BACE protein sequences. Sequences are labeled as in Table 2. Black dots indicate sequences with accession numbers for complete ORFs in Table 2. Solid vertical bars represent bootstrap values of $100 \%$ and open bars represent bootstrap values of $95 \%$ or greater but less than $100 \%$. Sequences corresponding to high order taxa are enclosed in colored boxes as indicated by the legends in the figures. (A): All sequences analyzed; (B): Ur-BACE sequences only. 


\begin{tabular}{|l|l|l||}
\hline Sequence ID & Length & Blocks \\
\hline Aipt_pall & 488 & \\
\hline Bran_flor & 493 & \\
\hline Capi_tela & 504 & \\
\hline Cion_inte & 466 & \\
\hline Cion_sauv & 458 & \\
\hline Clyt_hem & 474 & \\
\hline Cras_giga & 520 & \\
\hline Halo_rore & 463 & \\
\hline Lott_giga & 495 & \\
\hline Lymn_stagn & 544 & \\
\hline Nemo_vect & 479 & \\
\hline Plan_schmi & 516 & \\
\hline Schi_mans & 507 & \\
\hline Stron_purp & 538 & \\
\hline Tric_adhe_A & 545 & \\
\hline Tric_adhe_B & 505 & \\
\hline
\end{tabular}

FIGURE 4 | Blocks generated from the UrBACE sequences. The six conserved regions are shown in $\mathrm{N}$-terminal to $\mathrm{C}$-terminal order.

absolutely conserved in BACE1. While this suggests the palmitoylation could be pan-vertebrate, it raises the evolutionary question as to whether this is also a constitutive feature in BACE2 and/or the UrBACE. We explored the variation in the C-termini by using T-Coffee (Figure 7).

The results corroborate the very high conservation for the BACE1 C-terminal residues including the palmitoylated Cys candidates. It also indicates a possible single Cys candidate for BACE2 (at around 563 in the alignment) but this is absent in Xenopus and the gapping reflects low conservation. The difference for the UrBACEs is striking in that conservation falls off rapidly in the C-terminal cytoplasmic tail directly distal to the TM domain boundary. It can be noted here that the Strongylocentrotus purpuratus insert is confirmed by many ESTs (i.e., is not a gene prediction artifact). Significantly, a quartet of Cys candidates can be discerned, with the fourth one around position 615, despite the gapping in this region.

Notably, within the UrBACEs the block-type conservation coexists with variation in conservation patterns and length at the termini. We interpret the blocks as being directly associated (as part of the active site) or indirectly (as the core buried 3D fold sections) associated with catalysis. In contrast the signatures of the signal peptide and the CTM are physical property-based. They are thus not only less conserved as sequences per se but also less constrained in position (even though the CTM needs to be a defined membrane-spanning length) as they are surface features. This also explains why UrBACEs vary by up to 100 residues in length.

\section{Ka/Ks ANALYSIS}

The ratio of non-synonymous (protein sequence-changing) to synonymous (non-protein sequence-changing) mutation rates within a sequence, referred to as the $\mathrm{Ka} / \mathrm{Ks}$ ratio, is an indicator of the strength of purifying selection. A low ratio indicates strong purifying selection; a value close to 1 indicates no selection, and a value above 1 positive selection (adaptive change of the sequence). Analysis was carried out on complete cDNA sequences and individual exons to reveal any differential selection along the length of the gene. The sets that could be analyzed (i.e., cDNAs of equal length) had to be different between BACE1 and BACE2, because of the limited availability of complete mammalian sequences. For BACE1, sequences from H. sapiens, A. melanoleuca, S. scrofa. E. caballus, O. cuniculus, B. taurus, M. musculus, and R. norvergicus were analyzed. For BACE2, these were from $H$. sapiens, B. taurus, S. scrofa, M. musculus, $R$. norvegicus, and P. capensis.

The values presented in Table 3 are the overall mean values for the sequence set generated by SLAC (Kosakovsky Pond and Frost, 2005). For the whole genes, $\mathrm{Ka} / \mathrm{Ks}$ ratios were well below 1 , indicating a predominant role for purifying selection in the evolution of mammalian BACE genes. Values for BACE2 were generally higher (i.e., less stringent selection) than for BACE1. This is consistent with more rapid evolution of BACE2 seen in the phylogenetic analysis, although the datasets here are not directly comparable due to their different compositions. Analysis of individual exons showed weaker purifying selection acting on the $5^{\prime}$ (N-terminal) end of the gene, especially exon 1, while the central region of the gene showed low values (strongest selection) although the patterns differed between BACE1 and BACE2. Exon 8 of BACE2 in particular showed an elevated $\mathrm{Ka} / \mathrm{Ks}$ value.

The signal and pro-peptide domains showed elevated $\mathrm{Ka} / \mathrm{Ks}$. In BACE2, but not in BACE1, high $\mathrm{Ka} / \mathrm{Ks}$ was also observed for the C-terminal transmembrane domain. None of these $\mathrm{Ka} / \mathrm{Ks}$ values approached 1 , suggesting weaker purifying selection rather than positive selection when $\mathrm{Ka} / \mathrm{Ks}$ ratio were higher. This is not unexpected, given that these surface-exposed sections are constrained more by the more general physical residue properties for membrane interaction than sequence conservation per se. The fact that we noted lower $\mathrm{Ka} / \mathrm{Ks}$ in the BACE1 C-terminal may be related to the importance of the lipid anchoring features referred to above.

It should be noted that the Ka/Ks analysis is restricted to mammals as opposed to the deeper taxonomic levels dealt with via the larger protein sequence comparisons described in the previous section. However, the evolutionary insights they provide are complementary.

\section{PHYLOGENETIC DISTRIBUTION OF SUBSTRATES}

We tested for the presence of a small number of reportedly significant BACE1 and BACE2 human substrates in model organisms and selected phylum representatives. We included presenilin 1 (PSEN1) as a highly conserved component of the gamma secretase complex as a comparison. The BLAST results (see Supplementary Data file) are summarized in Figure 8.

The figure indicates discordant evolutionary trajectories between the BACEs and the human substrates. Given the long divergence times, where BLAST scores were low (e-5-e-8) it was difficult to discriminate between short domain matches (e.g., the disulphide-rich Kunitz domain in APP) and extended but low similarity scores indicating possible orthology. However, identity and match length were useful guides. We thus conclude that APP is absent from Trichoplax and Ciona, NRG1 and SCN2B are only 

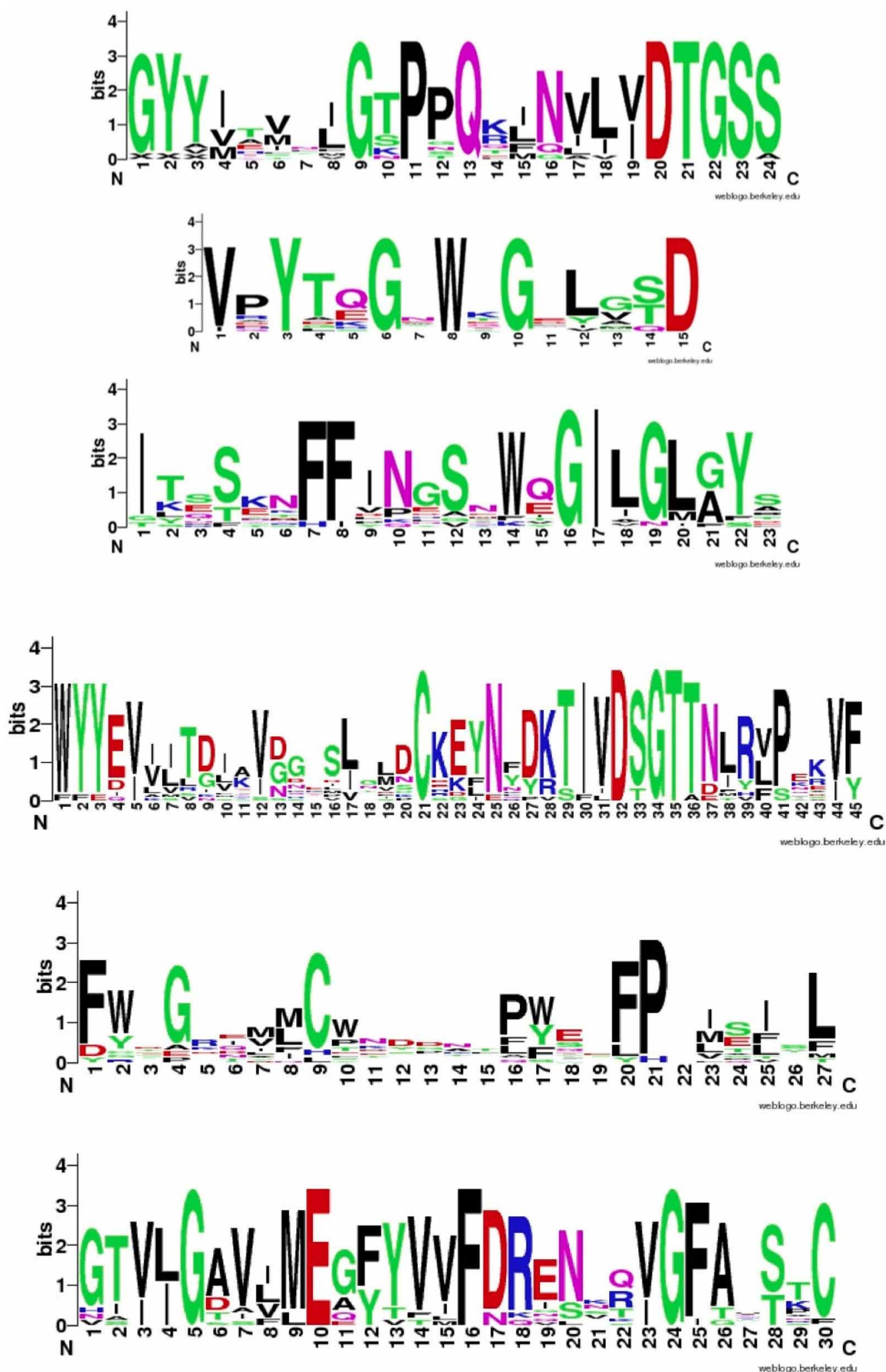

FIGURE 5 | Sequence Logos for the six conserved UrBACE blocks in N-terminal to C-terminal order (with spacing shown in Table 2). Residue letter height is an index of conservation. 


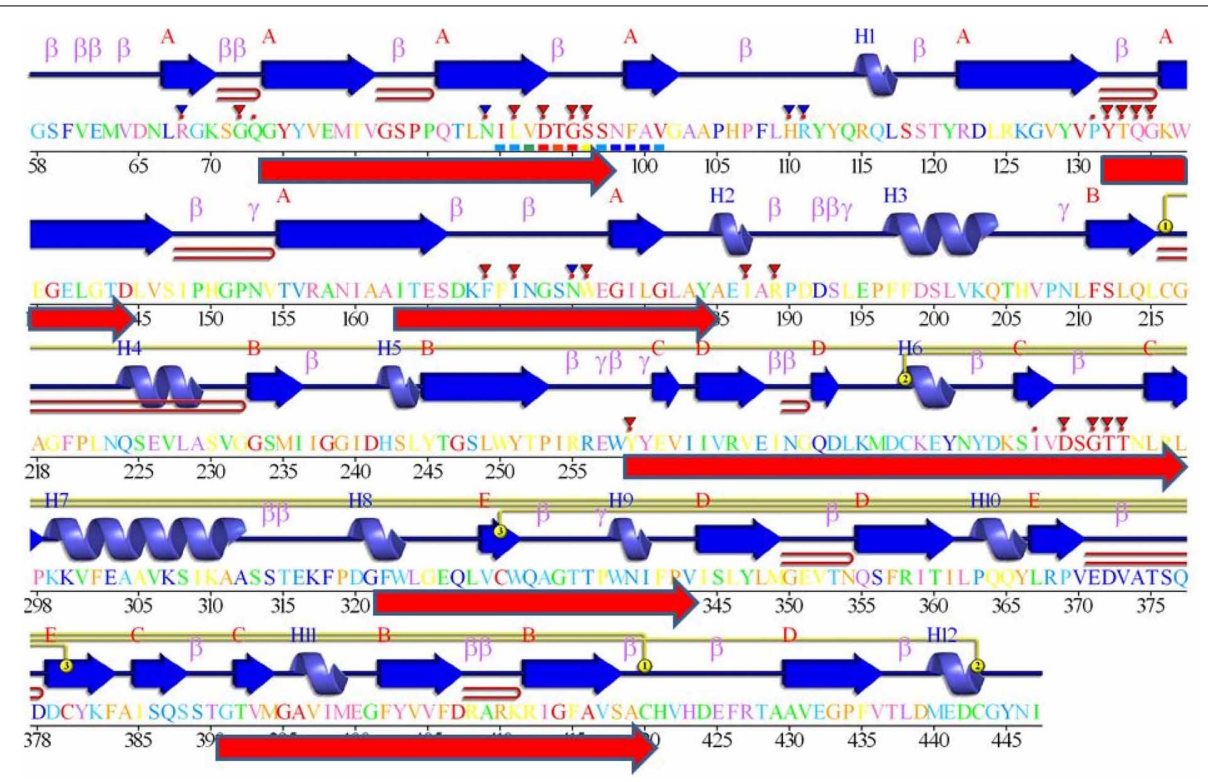

FIGURE 6 | The blocks from Figure 3 and Logos from Figure 4, aligned against a BACE1 PDBSum entry. Among the many entries with different ligands the longest sequence was chosen, 3lpi. The 2D display is shaded red to purple for conserved residues but note these are derived from BACE1 alignments (i.e., do not include UrBACEs). The secondary structure elements in dark blue and other marked features are described in the PDBSum features. The red arrows, added from this work, correspond to the six UrBACE sequence blocks. present in Zebrafish but TMEM27 is present only in Zebrafish and Ciona. In Drosophila and C. elegans we see the opposite case of APP homologs in the absence of BACE-like sequences.

\section{GENE EXPRESSION}

The consensus from many investigations is that BACE1 is predominantly a neuronal protein in humans, although glia cells may also produce significant amounts. However, systematic expression data is still sparse outside humans and mouse. We mined high-level expression patterns for BACEs using the Bgee resource (Bastian et al., 2008). While this collates expression data for human, mouse, zebrafish, Xenopus and Drosophila, coverage is patchy and difficult to standardize between ESTs and microarray probes. Expression patterns for BACE1 and BACE2 in human, mouse and Xenopus are compared in Table 4. Broadly, BACEs 1 and 2 showed similar expression patterns with a few exceptions. In human the only notable difference between them was that BACE2 was reported to be expressed in pancreas whereas BACE1 was not. In mouse, BACE2 was additionally expressed in the skin and prostate gland while BACE1 was not, and in the endocrine system BACE2 was restricted to the pineal gland whereas BACE1 was also expressed in the adrenal gland and neurohypophysis. In Xenopus, although both BACE1 and BACE2 were expressed in the testis they otherwise had distinct expression patterns: BACE1 was expressed in the brain while BACE2 was expressed in the oviduct, skin and spleen. No expression data were available for Zebrafish.

\section{DISCUSSION}

In this paper we have mined existing complete and partial genome sequences for new BACE gene families. Blending a range of resources by manual annotation produced a rich dataset, much of it not readily available from sequence databases. This allowed us to shed new light both on the evolutionary history of the BACE genes and on putative shifts in protein function and substrate interactions. We can consequently suggest future functional characterization making use of a range of model organisms that will be relevant to development of new therapies for Alzheimer's Disease and Type 2 Diabetes Mellitus.

\section{EVOLUTIONARY TRAJECTORIES}

Our sampling of most major phyla clearly delineates an "UrBACE." What we mean by this is the ancestral BACE-like sequence lineage with properties distinct from cathepsin-like precursors. The similarity scores and domain arrangement indicate that the emergence of this lineage was a distinct rather than gradual event and possibly related to the shuffling-in of a CTM domain into the $3^{\prime}$ exon. This would significantly change cellular trafficking and $\mathrm{pH}$ optima. We cannot detect any apparent intermediate form, such as a cathepsin with a CTM domain. By implication, these UrBACE sequences underwent selection for altered or new biochemical functions after duplication. This may have taken place before the origin of the choanoflagellates in the late Precambrian at least 860 MYA (million years ago) (Hedges et al., 2006; Blair, 2009).

Our results show that the Ur-BACE has a rich and previously undocumented history of gene duplication with preservation in different lineages. The most prominent is that leading to BACE1 and BACE2 after the divergence of the Hyperoartia (represented here by $P$. marinus) from the Gnathostomata (see Figure 3). This divergence, dated at c 530 MYA (Hedges et al., 2006; Blair, 2009), corresponds to the so-called 3R whole genome duplication (Holland et al., 1994; Miyata and Suga, 2001; Venkatesh et al., 2007; Hufton et al., 2008). This might have given rise to the paralogous and persistent BACE1 and BACE2 lineages, although 


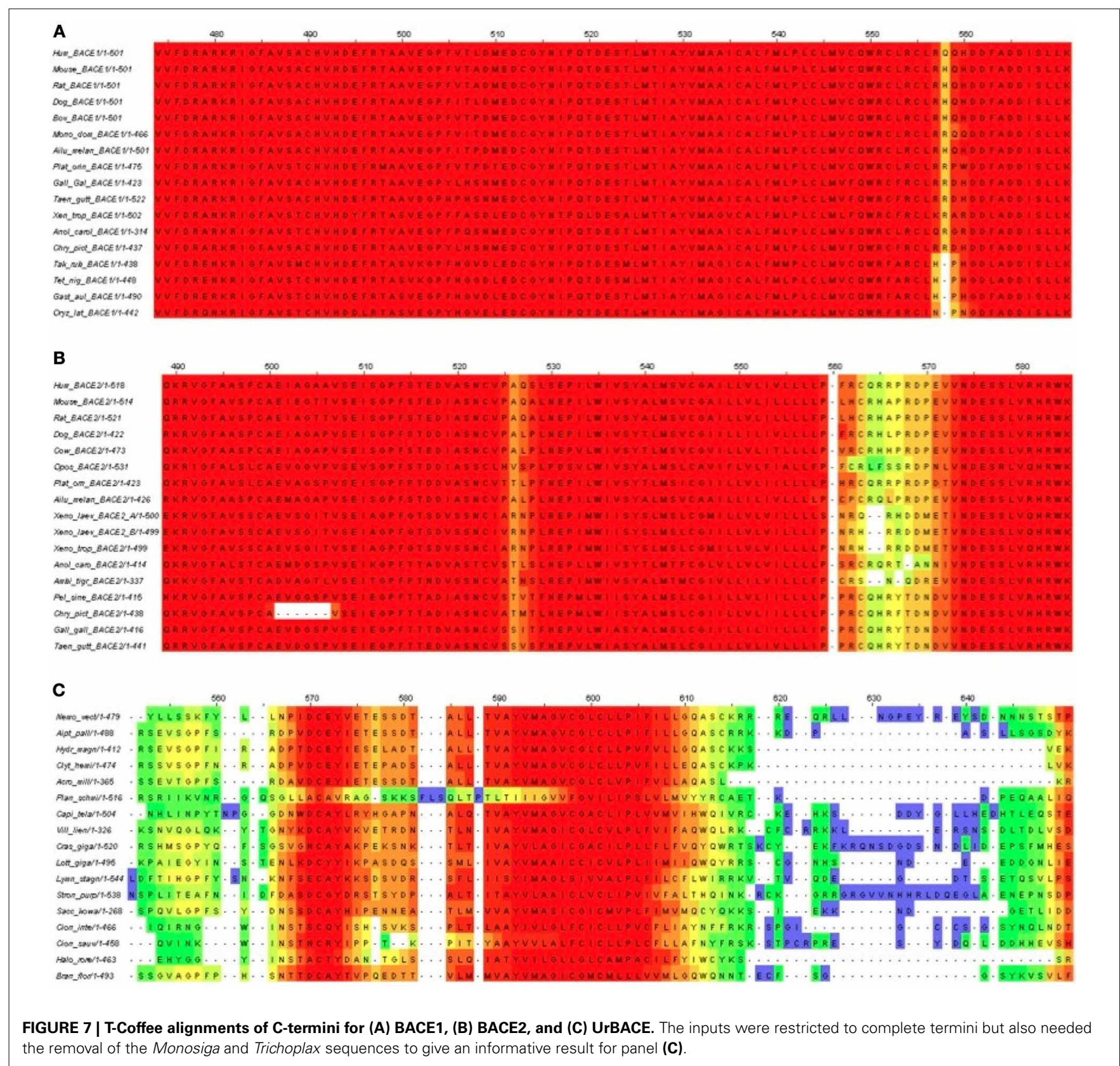

phylogenetic analysis of other gene families suggests that different scenarios are possible (Kuraku et al., 2009).

Our phylogeny deviates from current taxonomic groupings but it should be noted that it has the known constitutive limitations of a gene-specific analysis. In particular, we would have expected the Cnidaria to be more basal. However, much of this deviation reflects the poor resolution of this part of the phylogeny, which contains few groups supported by high (>95\%) bootstrap values. Despite this, the main taxonomic groups are mostly monophyletic except for the Protostomia. The clustering of the three Ciona UrBACEs away from the chordate lineage when we would have expected them to lie closest to B. floridae is also notable.
Gene duplication, whether or not it is associated with whole genome duplication, is a well-known process allowing the exploration of functional space by a gene family. It is thought that most duplicates are lost due to purifying selection or neutral drift soon after emergence, as they accumulate inactivating mutations (Lynch and Conery, 2000; Lynch and Force, 2000; Lynch et al., 2001). Long-term persistence of gene duplicates, as we have recorded here, is therefore prima facie evidence of both functional importance and differentiation in a gene family. This can be reflected in subfunctionalization (the redistribution of the ancestral gene's functions between its daughter genes) or neofunctionalization (the evolution of a new function by one of the duplicates) (Lynch and Force, 2000; Lynch et al., 2001). 
Table 3 | Results of $\mathrm{Ka} / \mathrm{Ks}$ analysis for subsections of BACE1 and BACE2 cDNA sequences.

\begin{tabular}{|c|c|c|c|c|}
\hline \multirow[t]{2}{*}{ Sequence } & \multicolumn{2}{|l|}{ BACE1 } & \multicolumn{2}{|l|}{ BACE2 } \\
\hline & $\begin{array}{l}\text { AA Coords } \\
\text { (Human protein) }\end{array}$ & $\mathrm{Ka} / \mathrm{Ks}$ & $\begin{array}{l}\text { AA Coords } \\
\text { (Human protein) }\end{array}$ & $\mathrm{Ka} / \mathrm{Ks}$ \\
\hline All & $1-501$ & 0.040 & $1-518$ & 0.144 \\
\hline \multicolumn{5}{|l|}{ DOMAINS } \\
\hline Signal & $1-21$ & 0.284 & $1-20$ & 0.156 \\
\hline Propeptide & $22-45$ & 0.127 & $21-62$ & 0.282 \\
\hline Chain & $46-501$ & 0.025 & $63-518$ & 0.126 \\
\hline Extracellular & $46-457$ & 0.025 & $63-473$ & 0.124 \\
\hline Transmembrane & $458-478$ & 0.000 & $474-494$ & 0.303 \\
\hline Intracellular & 479-501 & 0.053 & $495-518$ & 0.093 \\
\hline \multicolumn{5}{|l|}{ EXONS } \\
\hline Exon 1 & $1-87$ & 0.139 & $1-104$ & 0.216 \\
\hline Exon 2 & $88-117$ & 0.000 & $105-134$ & 0.105 \\
\hline Exon 3 & 118-189 & 0.014 & 135-206 & 0.060 \\
\hline Exon 4 & 190-235 & 0.032 & $207-249$ & 0.057 \\
\hline Exon 5 & $236-280$ & 0.007 & 250-294 & 0.040 \\
\hline Exon 6 & $281-314$ & 0.000 & $295-328$ & 0.059 \\
\hline Exon 7 & $315-364$ & 0.000 & $329-378$ & 0.027 \\
\hline Exon 8 & $365-422$ & 0.008 & $379-435$ & 0.211 \\
\hline Exon 9 & $423-501$ & 0.028 & $436-518$ & 0.140 \\
\hline
\end{tabular}

Just coding sequences were used (i.e., excluding untranslated regions). Values marked in bold exceed the overall value for the complete cDNAs.

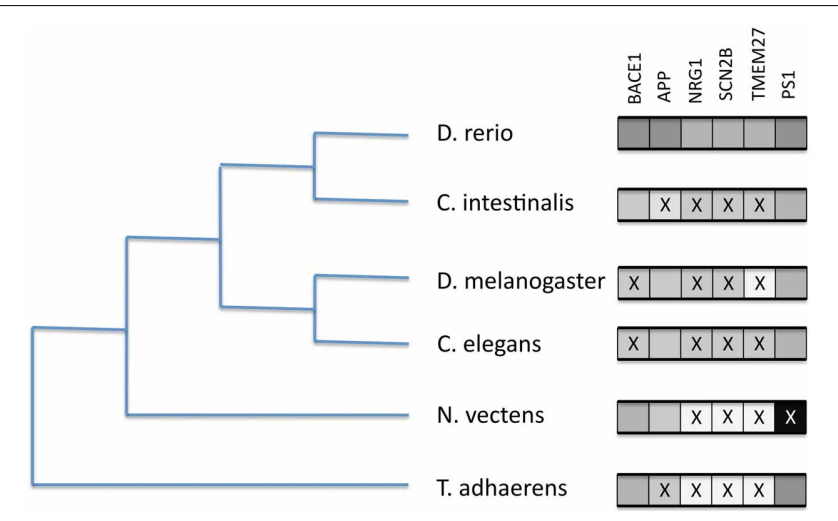

FIGURE 8 | Presence/absence of BACE1 and BACE substrates on six selected proteome sets from completed genomes. Boxes to the right represent likely orthology matches to the human sequences for BACE1 (P56817), APP (P05067), NRG1 (Q02297), SCN2B (O60939), TMEM27 (Q9HBJ8), and PSEN1 (P49768). An X represents their probable absence by low BLASTP score.

It has been suggested that duplicated genes undergo accelerated evolution immediately after duplication as they adapt to new functions and undergo a concomitant increase in $\mathrm{Ka} / \mathrm{Ks}$ (Lynch and Conery, 2000). Our analyses of branch lengths in the BACE1 and BACE2 families supports such an asymmetry in evolutionary rates leading to accelerated functional diversification of BACE2 with implied preservation of ancestral function in BACE1. Estimates of $\mathrm{Ka} / \mathrm{Ks}$ based on complete cDNAs from
Table 4 | Expression of BACE1 and BACE2 in human, mouse, and Xenopus.

\begin{tabular}{|c|c|c|c|c|c|c|}
\hline \multirow{2}{*}{$\begin{array}{l}\text { Anatomical } \\
\text { locus }\end{array}$} & \multicolumn{3}{|c|}{ BACE1 } & \multicolumn{3}{|c|}{ BACE2 } \\
\hline & Human & Mouse & Xenopus & Human & Mouse & Xenopus \\
\hline $\begin{array}{l}\text { Cardiovascular } \\
\text { system }\end{array}$ & Y & Y & & Y & Y & \\
\hline $\begin{array}{l}\text { Respiratory } \\
\text { system }\end{array}$ & Y & Y & & Y & Y & \\
\hline $\begin{array}{l}\text { Haematological } \\
\text { system }\end{array}$ & Y & & & Y & & \\
\hline $\begin{array}{l}\text { Lymphoreticular } \\
\text { system }\end{array}$ & Y & & & Y & & Spleen \\
\hline $\begin{array}{l}\text { Alimentary } \\
\text { system }\end{array}$ & Y & Y & & Y & Y & \\
\hline $\begin{array}{l}\text { Urogenital } \\
\text { system }\end{array}$ & Y & Y & Testis & Y & Y & $\begin{array}{l}\text { Testis } \\
\text { Oviduct }\end{array}$ \\
\hline $\begin{array}{l}\text { Endocrine } \\
\text { system }\end{array}$ & Y & Y & & Y & $\begin{array}{l}\text { Pineal } \\
\text { gland } \\
\text { only }\end{array}$ & \\
\hline $\begin{array}{l}\text { Musculoskeletal } \\
\text { system }\end{array}$ & Y & Y & & Y & Y & \\
\hline Dermal system & Y & & & Y & Y & Y \\
\hline $\begin{array}{l}\text { Nervous } \\
\text { system }\end{array}$ & Y & Y & Brain & Y & Y & \\
\hline Pancreas & $\mathrm{N}$ & & & Y & & \\
\hline $\begin{array}{l}\text { Sensory organ } \\
\text { system }\end{array}$ & & Y & & & Y & \\
\hline Prostate & & & & & Y & \\
\hline
\end{tabular}

Presence of expression is marked by $Y$ unless a more specific locus is mentioned. N, No expression is reported in contrast to BACE2.

a subset of mammalian species indicated higher values in BACE2 but no evidence of positive selection. This suggests relaxed purifying selection has acted on BACE2, which is consistent with functional diversification. Purifying selection on these proteins has nevertheless remained strong, implying important biological functions.

An anomaly in the scenario above is that the X. laevis whole genome duplication (produced by allotetraploidization circa 50 MYA) may have led to eventual loss of both BACE1s. This confounds the otherwise pan-vertebrate post-duplication persistence of the two paralogs. However, there is a caveat in that polyploid genomes are particularly difficult to assemble, so the "missing" BACE1(s) may yet be discovered in X. laevis. Notwithstanding, if their absence is confirmed, we make the following experimentally testable predictions. The first is an expression split in X. lae$v i s$ if one of the BACE2s has shifted into the BACE1 neuronal role. There is a precedent for this from a study showing differential expression of $14 \%$ of $X$. laevis paralogous pairs, indicative of polyploidy-related subfunctionalization (Morin et al., 2006). We consider the alternative scenario of substitution of the BACE1 roles by another protease to be less likely, since this would need a radical functional shift to occur in $X$. laevis over a relatively short evolutionary timescale, but this is also a testable prediction. 
Two loss-of-function fates can befall post-duplication enzyme copies. The first is decay to a non-translated pseudogene; the second is abrogation of catalytic function, typically due to mutations in the vicinity of the active site (i.e., the sequence is selectively maintained but for some non-proteolytic function). While neither of these can be ruled out for all individual species represented here (or their existence at a significant allelic frequency in certain populations) we suggest that, as a general case, neither of these processes has played a significant role in the evolutionary trajectory of the UrBACEs or the resulting persistence of the BACE paralogs in the vertebrates. Observations supporting this conjecture can be summarized as follows:

1. None of the 78 sequences showed frame shifts or premature stop codons characteristic of pseudogenes.

2. EST sampling or TSA virtual cDNA assembly serves as a useful proxy for protein expression (although pseudogenes can show low levels of transcription). While transcript coverage is patchy in UrBACE-containing phyla, in cases where EST numbers were high, we usually detected extensive ORF coverage. This was found to be full length for Acropora millepora, Strongylocentrotus purpuratus, and Ciona intestinalis. Even for less sampled species such as Schmidtea mediterranea we found ESTs covering the $5^{\prime}$ and $3^{\prime}$ of the cDNA (DN315134 and DN303898).

3. For vertebrates, 24 Unigene entries (i.e., clustered ESTs and mRNAs) for BACE1 and 26 for BACE2 can be found. This verifies gene transcription in a significant number of species.

4. In regard to the active site residues (equivalent to Asp93 and Asp289 in human BACE1) the proximal Asp was identifiable in 67 of 78 sequences and the distal Asp289 in 72 of 78 (regardless of whether the neighboring residues conformed to Prosite PS00141 or not). Cases where one of these was missing could be attributable to genomic assembly or gene prediction errors. Thus, pending cDNA verification for predicted ORFs, our data set contains no evidence of non-catalytic UrBACEs or BACEs.

There is a possible exception in the form of an extensive insertion in the Schistosoma japonicum gene sequence (Figure 4) which, if confirmed, suggests, despite the presence of two active site Asps, a severe disruption of folding. Consequent catalytic inactivity could also explain the anomalous branch length in the phylogenetic tree and could be associated with evolutionary drift into new functions for this parasite. We cannot rule out, and indeed might expect that additional ancient duplications in the UrBACE lineage could have resulted in pseudogenization of some copies (the alternative being complete deletion). However, completely finished genomes would be needed to detect such cryptic duplications.

\section{SUBSTRATE PATTERNS}

Our analysis of substrate co-occurrence is more of a preliminary assessment than a comprehensive analysis. It had to be limited, not only because the set of reported BACE substrates is continually expanding but also because of the scale of the analysis that would be needed to discern the evolutionary trajectory for all of them. Nevertheless, we show clear cases of phylogenetic discordance from mammalian protease/substrate pairings that could possibly have co-evolved. For example, Drosophila has an APP homolog but no Ur-BACE, while Ciona has an Ur-BACE but no APP homolog. We thus show that this type of investigation may provide an indication of significant role shifts occurring between species over long divergence times. The automated GeneTree feature in Ensembl can also be used to follow the evolution of any selected substrate (with the caveat that coverage of basal phyla is more limited than we explore here).

\section{PUTATIVE Ur-BACE FUNCTIONS}

The minimum evolutionary model for the BACE gene family is that the UrBACE emerged at the base of the metazoan tree via the duplication of a cathepsin and the shuffling-in of the C-terminal TM domain. This is particularly significant because it thereby joins the limited number of proteases participating in regulated intramembrane proteolysis (RIP) of which APP beta-secretase is the archetype (Lal and Caplan, 2011; Lichtenthaler et al., 2011). Whether the Placozoans are basal to the Choanoflagellates in phylogenetic terms as urmetazoans, or vice versa, is still a subject for debate (Osigus et al., 2013). However, we detected further duplication, divergence and maintenance of the resulting paralogs in these smallest known urmetazoan gene repertoires [e.g., Monosiga brevicollis and Trichoplax adherens have only 9200 and 11,500 proteins, respectively (King et al., 2008; Srivastava et al., 2008)]. This argues for functional importance, as does their persistence for the subsequent $\sim 800$ million years. The absence of human-BACE substrates in organisms with one or more Ur-BACE sequences suggests these ancestral enzymes have different substrates and roles to those in humans. However, their loss in Ecdysozoans implies they can be functionally substituted by other proteases.

Considerations of possible ancestral functions based on bioinformatic evidence alone are speculative. Nevertheless, by extrapolating from mammalian BACE1 (which has greater similarity to Ur-BACE sequences than BACE2) we can suggest the Ur-BACEs might also have a neuronal role. While this would fit with our detection of the enzyme at the base of Bilateria (with primitive nervous systems) and the Cnidaria (with nerve nets), the counter argument would be that Placozoans and Choanoflagellates have no synaptically connected neurons. However, the surprise from the draft genomes of members of these phyla was that they not only showed extensive human protein homology but also similar domain combinations. The recent report that these phyla both express sodium channels included the suggestion that the evolution of these predates the origin of nervous systems (Liebeskind et al., 2011; Zakon, 2012). An independent observation echoes this in detecting a primordial neurosecretory apparatus in a choanoflagellate, identified via a SNARE protein complex (Burkhardt et al., 2011). Notably, A. queenslandica neither contains $\mathrm{Na}(\mathrm{v})$-like channels nor an UrBACE while BACE1 regulates $\mathrm{Na}(\mathrm{v}) 1$ channels in mouse neuronal cells by sequential processing of the SCN2B regulatory subunit (Kovacs et al., 2010). The question thus arises as to whether the UrBACE co-evolved for ion channel processing as one of its earliest functions after it acquired the CTM and RIP potential but this will also have to await experimental testing. These lines of evidence suggest that 
some of the molecular repertoire for multi-cellularity and signaling preceded the appearance of the nervous system and were later co-opted for their evolution.

The presumed functional divergence after duplication of BACEs points toward BACE1 maintaining the primary function of the UrBACE, or at least overlapping more closely with it than BACE2. However, intriguing implications arise from the recently reported functions of BACE2. One of these is the observation that pancreatic $\beta$-cells have many characteristics in common with nerve cells and may thus share with them a common evolutionary origin via gut cells co-opting a neuronal transcriptional program (Arntfield and van der Kooy, 2011). For example, while Hagfish and Lampreys have a pancreas made up almost entirely of $\beta$-cells, in Amphioxus these cells are associated with the intestinal tissue in a dispersed form (Pieler and Chen, 2006). An additional implication of an ancestral neuronal connection for BACE2 is that melanophore pigmentation cells are developmentally derived from the neural crest (Quigley et al., 2004). We can thus postulate that BACE2 was co-opted initially in a neuronal context (e.g., via the post-duplication gene dosage advantage) but later became selected for functions no longer confined to neural cells per se (i.e., neofunctionalization).

\section{FUTURE WORK}

Experimental characterization of ancestral BACE sequences, aided by the results presented, here, should have two important and related facets. The first is the purely scientific focus on biochemical and physiological roles for these enzymes in the complex degradomic protease webs that can now be compared between different phyla (auf dem Keller et al., 2007). Together with further bioinformatics analysis of basal eumetazoans, such work will reveal hitherto unknown roles, including the processing of new substrates that could have important implications for human BACE1 and BACE2. In addition, standardized comparative expression patterns across a wider variety of species will provide insight into pre- and post-gene duplication tissue specificity within and between taxa. Characterization of UrBACE(s) and putative homologous substrates may give more interpretable phenotypic and omics-profiling signatures compared to organisms with more complex gene repertoires. Some species with UrBACE sequences already have established experimental functional genomics platforms. For example, Ciona is used for studying chordate central nervous system regeneration (Dahlberg et al., 2009). These investigations will not only provide new testable hypotheses but also analogous putative substrate studies in C. elegans and Drosophila (UrBACE-negative organisms) could provide informative controls.

The second facet of further research relates to the crucial status of these enzymes as drug targets. This pair is unusual in being catalytically similar but the very different therapeutic indications in different tissues have been validated about a decade apart. Given the huge and increasing scale of unmet medical need for both $\mathrm{AD}$ and $\mathrm{T} 2 \mathrm{DM}$, progression toward clinical candidates for either target is to be earnestly hoped for. However, such firstin-class inhibitors face significant hurdles. Those directed against BACE1 will need (1) high dosing to cross the blood-brain barrier, (2) probably require life-long treatment, and (3) will need to be administered early to patients who are asymptomatic but with a robustly predictive biomarker profile (Karran et al., 2011). Under such circumstances, any new aspects of BACE biochemistry, implied or directly revealed by functional genomics, are going to be important. This would not only be in a predictive context for efficacy, toxicology and side effects, but also because new substrates can provide candidate biomarkers for clinical evaluation. While studies in mice and Zebrafish could be deemed potentially most informative, there is no phylogenetic grouping where experimental results could be considered irrelevant.

While BACE2 inhibition for T2DM has a longer way to go before clinical proof-of-concept, a review of recent patent publications indicates pharmaceutical company discovery programs for this indication have been running since 2010 (Southan, 2013). The major factors that might accelerate development are (1) the large collection of BACE1 inhibitor structures in PDB, (2) the collective experience in their optimization that can provide starting points for BACE2 inhibitors, and (3) unlike for BACE1, compounds need not be brain penetrant. Two years ago the numbers of compounds with inhibition results published in patents and papers was 5459 and 414 for human BACE1 and BACE2 respectively [supplementary data from (Southan et al., 2011)]. The BACE2 results were entirely from cross-screening of BACE1directed inhibitors. The equivalent inhibitor structure ratio from published papers in ChEMBL (release 16, June 2013) is now 3815 to 527, suggesting increased interest in BACE2.

The potential importance of new insights revealed by functional genomics applies equally for both enzymes, especially since the double-target status poses important new specificity questions. In the hitherto absence of predictable in vivo consequences of BACE2 inhibition, the specificity ratio (i.e., the BACE1:BACE2, IC50, or Ki) for a BACE1 lead compound was probably set empirically at $\sim 100$-fold. Development projects will now need to be more circumspect on how they choose this ratio; including monitoring for effects on the turnover of BACE2 substrates and $\beta$-cell function, as well as reciprocally monitoring BACE2 inhibitors for effects on the turnover of BACE1 substrates.

Our results have additional utility for chemical biology where these pure and applied facets intersect. For example, BACE inhibitors specific for either or both paralogs could be used as system probes in the way that inhibitors have already been used for mouse and fish experiments. This is analogous to genomic lossof-function but with the inherent advantages of small-molecule perturbation (e.g., rapid onset, precise dosing, mixture testing, analog testing, developmental stage specificity and reversal by wash-out). These can be informative when compared with activity ablation by RNAi, mutation or knockout. Such chemical perturbations could be tried out in basal phyla where we have identified Ur-BACEs with local active-site sequence similarities of $50 \%$ or above. Results are likely to be relevant to both the $\mathrm{AD}$ and T2DM drug discovery efforts. They could also lead to new types of BACE inhibitor cross-screens utilizing the moderate throughput phenotypic read-outs that these basal organisms can offer.

\section{AUTHOR CONTRIBUTIONS}

Christopher Southan initiated the study, extracted sequences, analyzed data, and contributed to the writing. John M. Hancock 
analyzed data and contributed to the writing. Both authors have approved the final version of the manuscript.

\section{ACKNOWLEDGMENTS}

We thank the referees for useful comments that enhanced the final version.

\section{FUNDING}

For the latter part of this work Christopher Southan was supported by Biomedical Resources Grant [099156/Z/12/Z] from the Wellcome Trust.

\section{SUPPLEMENTARY MATERIAL}

The Supplementary Material for this article can be found online at: http://www.frontiersin.org/journal/10.3389/fgene. 2013.00293/abstract

\section{REFERENCES}

Acquati, F., Accarino, M., Nucci, C., Fumagalli, P., Jovine, L., Ottolenghi, S., et al. (2000). The gene encoding DRAP (BACE2), a glycosylated transmembrane protein of the aspartic protease family, maps to the down critical region. FEBS Lett. 468, 59-64. doi: 10.1016/S0014-5793(00)01192-3

Anwar, N., and Hunt, E. (2009). Improved data retrieval from TreeBASE via taxonomic and linguistic data enrichment. BMC Evol. Biol. 9:93. doi: 10.1186/14712148-9-93

Arntfield, M. E., and van der Kooy, D. (2011). $\beta$-Cell evolution: How the pancreas borrowed from the brain: the shared toolbox of genes expressed by neural and pancreatic endocrine cells may reflect their evolutionary relationship. Bioessays 33, 582-587. doi: 10.1002/bies.201100015

Attwood, T. K., Coletta, A., Muirhead, G., Pavlopoulou, A., Philippou, P. B., Popov, I., et al. (2012). The PRINTS database: a fine-grained protein sequence annotation and analysis resource-its status in 2012. Database 2012:bas019. doi: 10.1093/database/bas019

auf dem Keller, U., Doucet, A., and Overall, C. M. (2007). Protease research in the era of systems biology. Biol. Chem. 388, 1159-1162. doi: 10.1515/BC.2007.146

Bastian, F., Parmentier, G., Roux, J., Moretti, S., Laudet, V., and Robinson-Rechavi, M. (2008). "Bgee: integrating and comparing heterogeneous transcriptome data among species," in Data Integration in the Life Sciences Lecture Notes in Computer Science, Vol. 5109, eds A. Bairoch, S. Cohen-Boulakia, and C. Froidevaux (Berlin: Springer), 124-131.

Blair, J. (2009). “Animals (Metazoa)," in The Timetree of Life, eds S. B. Hedges and S. Kumar (Oxford: Oxford University Press), 223-230.

Burkhardt, P., Stegmann, C. M., Cooper, B., Kloepper, T. H., Imig, C., Varoqueaux, F., et al. (2011). Primordial neurosecretory apparatus identified in the choanoflagellate Monosiga brevicollis. Proc. Natl. Acad. Sci. U.S.A. 108, 15264-15269. doi: 10.1073/pnas.1106189108

Carmine-Simmen, K., Proctor, T., Tschäpe, J., Poeck, B., Triphan, T., Strauss, R., et al. (2009). Neurotoxic effects induced by the Drosophila amyloid-beta peptide suggest a conserved toxic function. Neurobiol. Dis. 33, 274-281. doi: 10.1016/j.nbd.2008.10.014

Crooks, G. E., Hon, G., Chandonia, J.-M., and Brenner, S. E. (2004). WebLogo: a sequence logo generator. Genome Res. 14, 1188-1190. doi: 10.1101/gr.849004

Dahlberg, C., Auger, H., Dupont, S., Sasakura, Y., Thorndyke, M., and Joly, J.-S. (2009). Refining the Ciona intestinalis model of central nervous system regeneration. PLoS ONE 4:e4458. doi: 10.1371/journal.pone.0004458

Dislich, B., and Lichtenthaler, S. F. (2012). The membrane-bound aspartyl protease BACE1: molecular and functional properties in Alzheimer's disease and beyond. Front. Physiol. 3:8. doi: 10.3389/fphys.2012.00008

Dominguez, D., Tournoy, J., Hartmann, D., Huth, T., Cryns, K., Deforce, S., et al. (2005). Phenotypic and biochemical analyses of BACE1- and BACE2-deficient mice. J. Biol. Chem. 280, 30797-30806. doi: 10.1074/jbc.M505249200

Drew, B. T. (2013). Data deposition: missing data mean holes in tree of life. Nature 493, 305. doi: 10.1038/493305f

Durham, T. B., and Shepherd, T. A. (2006). Progress toward the discovery and development of efficacious BACE inhibitors. Curr. Opin. Drug Discov. Devel. 9, 776-791.
Esterhazy, D., Stutzer, I., Wang, H., Rechsteiner, M. P., Beauchamp, J., Dobeli, H., et al. (2011). Bace2 is a beta cell-enriched protease that regulates pancreatic beta cell function and mass. Cell Metab. 14, 365-377. doi: 10.1016/j.cmet.2011.06.018

Farzan, M., Schnitzler, C. E., Vasilieva, N., Leung, D., and Choe, H. (2000). BACE2, a beta -secretase homolog, cleaves at the beta site and within the amyloid-beta region of the amyloid-beta precursor protein. Proc. Natl. Acad. Sci. U.S.A. 97, 9712-9717. doi: 10.1073/pnas.160115697

Fleck, D., Garratt, A. N., Haass, C., and Willem, M. (2012). BACE1 dependent neuregulin proteolysis. Curr. Alzheimer Res. 9, 178-183. doi: 10.2174/ 156720512799361637

Gascuel, O. (1997). BIONJ: an improved version of the NJ algorithm based on a simple model of sequence data. Mol. Biol. Evol. 14, 685-695. doi: 10.1093/oxfordjournals.molbev.a025808

Goedert, M., and Spillantini, M. G. (2006). A century of Alzheimer's disease. Science 314, 777-781. doi: 10.1126/science. 1132814

Gonnet, G. H. (2012). Surprising results on phylogenetic tree building methods based on molecular sequences. BMC Bioinformatics 13:148. doi: 10.1186/14712105-13-148

Hedges, S. B., Dudley, J., and Kumar, S. (2006). TimeTree: a public knowledgebase of divergence times among organisms. Bioinformatics 22, 2971-2972. doi: 10.1093/bioinformatics/btl505

Hemming, M. L., Elias, J. E., Gygi, S. P., and Selkoe, D. J. (2009). Identification of beta-secretase (BACE1) substrates using quantitative proteomics. PLoS ONE 4:e8477. doi: 10.1371/journal.pone.0008477

Henikoff, S., Henikoff, J. G., Alford, W. J., and Pietrokovski, S. (1995). Automated construction and graphical presentation of protein blocks from unaligned sequences. Gene 163, GC17-GC26. doi: 10.1016/0378-1119(95)00486-P

Hilpert, H., Guba, W., Woltering, T. J., Wostl, W., Pinard, E., Mauser, H., et al. (2013). $\beta$-Secretase (BACE1) inhibitors with high in vivo efficacy suitable for clinical evaluation in Alzheimer's disease. J. Med. Chem. 56, 3980-3995. doi: 10.1021/jm400225m

Hogl, S., Kuhn, P. H., Colombo, A., and Lichtenthaler, S. F. (2011). Determination of the proteolytic cleavage sites of the amyloid precursor-like protein 2 by the proteases ADAM10, BACE1 and gamma-secretase. PLoS ONE 6:e21337. doi: 10.1371/journal.pone.0021337

Hogl, S., van Bebber, F., Dislich, B., Kuhn, P.-H., Haass, C., Schmid, B., et al. (2013). Label-free quantitative analysis of the membrane proteome of Bacel protease knock-out zebrafish brains. Proteomics 13, 1519-1527. doi: 10.1002/pmic.201200582

Holland, P. W., Garcia-Fernandez, J., Williams, N. A., and Sidow, A. (1994). Gene duplications and the origins of vertebrate development. Dev. Suppl. Gene duplications and the origins of vertebrate development, 125-133.

Hufton, A. L., Groth, D., Vingron, M., Lehrach, H., Poustka, A. J., and Panopoulou, G. (2008). Early vertebrate whole genome duplications were predated by a period of intense genome rearrangement. Genome Res. 18, 1582-1591. doi: 10.1101/gr.080119.108

Hussain, I., Powell, D., Howlett, D. R., Tew, D. G., Meek, T. D., Chapman, C., et al. (1999). Identification of a novel aspartic protease (Asp 2) as beta-secretase. Mol. Cell. Neurosci. 14, 419-427. doi: 10.1006/mcne.1999.0811

Hussain, I., Powell, D. J., Howlett, D. R., Chapman, G. A., Gilmour, L., Murdock, P. R., et al. (2000). ASP1 (BACE2) cleaves the amyloid precursor protein at the beta-secretase site. Mol. Cell. Neurosci. 16, 609-619. doi: 10.1006/mcne.2000.0884

Jonsson, T., Atwal, J. K., Steinberg, S., Snaedal, J., Jonsson, P. V., Bjornsson, S., et al. (2012). A mutation in APP protects against Alzheimer's disease and age-related cognitive decline. Nature 488, 96-99. doi: 10.1038/nature11283

Karran, E., Mercken, M., and De Strooper, B. (2011). The amyloid cascade hypothesis for Alzheimer's disease: an appraisal for the development of therapeutics. Nat. Rev. Drug Discov. 10, 698-712. doi: 10.1038/nrd3505

King, N., Westbrook, M. J., Young, S. L., Kuo, A., Abedin, M., Chapman, J., et al. (2008). The genome of the choanoflagellate Monosiga brevicollis and the origin of metazoans. Nature 451, 783-788. doi: 10.1038/nature06617

Kosakovsky Pond, S. L., and Frost, S. D. W. (2005). Not so different after all: a comparison of methods for detecting amino acid sites under selection. Mol. Biol. Evol. 22, 1208-1222. doi: 10.1093/molbev/msil05

Kovacs, D. M., Gersbacher, M. T., and Kim, D. Y. (2010). Alzheimer's secretases regulate voltage-gated sodium channels. Neurosci. Lett. 486, 68-72. doi: 10.1016/j.neulet.2010.08.048 
Kuhn, P.-H., Koroniak, K., Hogl, S., Colombo, A., Zeitschel, U., Willem, M., et al. (2012). Secretome protein enrichment identifies physiological BACE1 protease substrates in neurons. EMBO J. 31, 3157-3168. doi: 10.1038/emboj. 2012.173

Kuraku, S., Meyer, A., and Kuratani, S. (2009). Timing of genome duplications relative to the origin of the vertebrates: did cyclostomes diverge before or after? Mol. Biol. Evol. 26, 47-59. doi: 10.1093/molbev/msn222

Lal, M., and Caplan, M. (2011). Regulated intramembrane proteolysis: signaling pathways and biological functions. Physiology 26, 34-44. doi: 10.1152/physiol.00028.2010

Laskowski, R. A. (2009). PDBsum new things. Nucleic Acids Res. 37, D355-D359. doi: $10.1093 / \mathrm{nar} / \mathrm{gkn} 860$

Leebens-Mack, J., Vision, T., Brenner, E., Bowers, J. E., Cannon, S., Clement, M. J., et al. (2006). Taking the first steps towards a standard for reporting on phylogenies: Minimum Information About a Phylogenetic Analysis (MIAPA). Omics 10, 231-237. doi: 10.1089/omi.2006.10.231

Lichtenthaler, S. F., Haass, C., and Steiner, H. (2011). Regulated intramembrane proteolysis-lessons from amyloid precursor protein processing. J. Neurochem. 117, 779-796. doi: 10.1111/j.1471-4159.2011.07248.x

Liebeskind, B. J., Hillis, D. M., and Zakon, H. H. (2011). Evolution of sodium channels predates the origin of nervous systems in animals. Proc. Natl. Acad. Sci. U.S.A. 108, 9154-9159. doi: 10.1073/pnas.1106363108

Löytynoja, A., and Goldman, N. (2010). webPRANK: a phylogeny-aware multiple sequence aligner with interactive alignment browser. BMC Bioinformatics 11:579. doi: 10.1186/1471-2105-11-579

Lynch, M., and Conery, J. S. (2000). The evolutionary fate and consequences of duplicate genes. Science 290, 1151-1155. doi: 10.1126/science.290.5494.1151

Lynch, M., and Force, A. (2000). The probability of duplicate gene preservation by subfunctionalization. Genetics 154, 459-473.

Lynch, M., O'Hely, M., Walsh, B., and Force, A. (2001). The probability of preservation of a newly arisen gene duplicate. Genetics 159, 1789-1804.

Ma, H., Lesne, S., Kotilinek, L., Steidl-Nichols, J. V., Sherman, M., Younkin, L., et al. (2007). Involvement of beta-site APP cleaving enzyme 1 (BACE1) in amyloid precursor protein-mediated enhancement of memory and activitydependent synaptic plasticity. Proc. Natl. Acad. Sci. U.S.A. 104, 8167-8172. doi: 10.1073/pnas.0609521104

May, P. C., Dean, R. A., Lowe, S. L., Martenyi, F., Sheehan, S. M., Boggs, L. N., et al. (2011). Robust central reduction of amyloid- $\beta$ in humans with an orally available, non-peptidic $\beta$-secretase inhibitor. J. Neurosci. 31, 16507-16516. doi: 10.1523/JNEUROSCI.3647-11.2011

Miyata, T., and Suga, H. (2001). Divergence pattern of animal gene families and relationship with the Cambrian explosion. Bioessays 23, 1018-1027. doi: 10.1002/bies.1147

Morin, R. D., Chang, E., Petrescu, A., Liao, N., Griffith, M., Chow, W., et al. (2006). Sequencing and analysis of 10,967 full-length cDNA clones from Xenopus laevis and Xenopus tropicalis reveals post-tetraploidization transcriptome remodeling. Genome Res. 16, 796-803. doi: 10.1101/gr.4871006

Olson, R. E., and Albright, C. F. (2008). Recent progress in the medicinal chemistry of gamma-secretase inhibitors. Curr. Top. Med. Chem. 8, 17-33. doi: $10.2174 / 156802608783334088$

Osigus, H.-J., Eitel, M., and Schierwater, B. (2013). Chasing the urmetazoon: striking a blow for quality data? Mol. Phylogenet. Evol. 66, 551-557. doi: 10.1016/j.ympev.2012.05.028

Papadopoulos, J. S., and Agarwala, R. (2007). COBALT: constraint-based alignment tool for multiple protein sequences. Bioinformatics 23, 1073-1079. doi: 10.1093/bioinformatics/btm076

Pieler, T., and Chen, Y. (2006). Forgotten and novel aspects in pancreas development. Biol. Cell 98, 79-88. doi: 10.1042/BC20050069

Probst, G., and Xu, Y. (2012). Small-molecule BACE1 inhibitors: a patent literature review (2006 - 2011). Expert Opin. Ther. Pat. 22, 511-540. doi: $10.1517 / 13543776.2012 .681302$

Pruitt, K. D., Harrow, J., Harte, R. A., Wallin, C., Diekhans, M., Maglott, D. R., et al. (2009). The consensus coding sequence (CCDS) project: Identifying a common protein-coding gene set for the human and mouse genomes. Genome Res. 19, 1316-1323. doi: 10.1101/gr.080531.108

Quigley, I. K., Turner, J. M., Nuckels, R. J., Manuel, J. L., Budi, E. H., MacDonald, E. L., et al. (2004). Pigment pattern evolution by differential deployment of neural crest and post-embryonic melanophore lineages in Danio fishes. Development 131, 6053-6069. doi: 10.1242/dev.01526
Rausch, T., Emde, A.-K., Weese, D., Döring, A., Notredame, C., and Reinert, K. (2008). Segment-based multiple sequence alignment. Bioinformatics 24, i187-i192. doi: 10.1093/bioinformatics/btn281

Rawlings, N. D., and Bateman, A. (2009). Pepsin homologues in bacteria. BMC Genomics 10:437. doi: 10.1186/1471-2164-10-437

Rawlings, N. D., Morton, F. R., Kok, C. Y., Kong, J., and Barrett, A. J. (2008). MEROPS: the peptidase database. Nucleic Acids Res. 36, D320-D325. doi: 10.1093/nar/gkm954

Rochin, L., Hurbain, I., Serneels, L., Fort, C., Watt, B., Leblanc, P., et al. (2013). BACE2 processes PMEL to form the melanosome amyloid matrix in pigment cells. Proc. Natl. Acad. Sci. U.S.A. 110, 10658-10663. doi: 10.1073/pnas. 1220748110

Ruan, J., Li, H., Chen, Z., Coghlan, A., Coin, L. J. M., Guo, Y., et al. (2008). TreeFam: 2008 Update. Nucleic Acids Res. 36, D735-D740. doi: 10.1093/nar/gkm1005

Schechter, I., and Ziv, E. (2011). Cathepsins, S., B and L with aminopeptidases display $\beta$-secretase activity associated with the pathogenesis of Alzheimer's disease. Biol. Chem. 392, 555-569. doi: 10.1515/bc.2011.054

Selkoe, D. J., and Wolfe, M. S. (2007). Presenilin: running with scissors in the membrane. Cell 131, 215-221. doi: 10.1016/j.cell.2007.10.012

Shaffer, H. B., Minx, P., Warren, D. E., Shedlock, A. M., Thomson, R. C., Valenzuela, N., et al. (2013). The western painted turtle genome, a model for the evolution of extreme physiological adaptations in a slowly evolving lineage. Genome Biol. 14:R28. doi: 10.1186/gb-2013-14-3-r28

Singh, J. (2011). FigShare. J. Pharmacol. Pharmacother. 2, 138-139. doi: 10.4103/0976-500X.81919

Sinha, S., Anderson, J. P., Barbour, R., Basi, G. S., Caccavello, R., Davis, D., et al. (1999). Purification and cloning of amyloid precursor protein beta-secretase from human brain. Nature 402, 537-540. doi: 10.1038/990114

Southan, C. (2007). Exploiting new genome data and Internet resources for the phylogenetic analysis of proteases, substrates and inhibitors. Biochem. Soc. Trans. 35, 599-603. doi: 10.1042/BST0350599

Southan, C. (2013). BACE2 as a new diabetes target: a patent review (2010 - 2012). Expert Opin. Ther. Pat. 23, 649-663. doi: 10.1517/13543776.2013.780032

Southan, C., Boppana, K., Jagarlapudi, S. A., and Muresan, S. (2011). Analysis of in vitro bioactivity data extracted from drug discovery literature and patents: ranking 1654 human protein targets by assayed compounds and molecular scaffolds. J. Cheminform. 3, 14. doi: 10.1186/1758-2946-3-14

Srivastava, M., Begovic, E., Chapman, J., Putnam, N. H., Hellsten, U., Kawashima, T., et al. (2008). The Trichoplax genome and the nature of placozoans. Nature 454, 955-960. doi: 10.1038/nature07191

Stamford, A. W., Scott, J. D., Li, S. W., Babu, S., Tadesse, D., Hunter, R., et al. (2012). Discovery of an orally available, brain penetrant BACE1 inhibitor that affords robust CNS A $\beta$ Reduction. ACS Med. Chem. Lett. 3, 897-902. doi: $10.1021 / \mathrm{ml} 3001165$

Stockley, J. H., and O'Neill, C. (2008). Understanding BACE1: essential protease for amyloid-beta production in Alzheimer's disease. Cell. Mol. Life Sci. 65, 3265-3289. doi: 10.1007/s00018-008-8271-3

Stoltzfus, A., O’Meara, B., Whitacre, J., Mounce, R., Gillespie, E. L., Kumar, S., et al. (2012). Sharing and re-use of phylogenetic trees (and associated data) to facilitate synthesis. BMC Res. Notes 5:574. doi: 10.1186/1756-05005-574

Stützer, I., Selevsek, N., Esterházy, D., Schmidt, A., Aebersold, R., and Stoffel, M. (2013). Systematic proteomic analysis identifies $\beta$-site amyloid precursor protein cleaving enzyme 2 and 1 (BACE2 and BACE1) substrates in pancreatic $\beta$-cells. J. Biol. Chem. 288, 10536-10547. doi: 10.1074/jbc.M112. 444703

Sun, X., He, G., and Song, W. (2006). BACE2, as a novel APP theta-secretase, is not responsible for the pathogenesis of Alzheimer's disease in Down syndrome. FASEB J. 20, 1369-1376. doi: 10.1096/fj.05-5632com

Sun, X., Wang, Y., Qing, H., Christensen, M. A., Liu, Y., Zhou, W., et al. (2005). Distinct transcriptional regulation and function of the human BACE2 and BACE1 genes. FASEB J. 19, 739-749. doi: 10.1096/fj.04-3426com

Toyn, J. H., Lin, X.-A., Thompson, M. W., Guss, V., Meredith, J. E., Sankaranarayanan, S., et al. (2010). Viable mouse gene ablations that robustly alter brain $A \beta$ levels are rare. BMC Neurosci. 11:143. doi: 10.1186/1471-220211-143

Van Bebber, F., Hruscha, A., Willem, M., Schmid, B., and Haass, C. (2013). Loss of Bace 2 in zebrafish affects melanocyte migration and is distinct from Bacel knock out phenotypes. J. Neurochem. 127, 471-481. doi: 10.1111/jnc.12198 
Vassar, R., Bennett, B. D., Babu-Khan, S., Kahn, S., Mendiaz, E. A., Denis, P., et al. (1999). Beta-secretase cleavage of Alzheimer's amyloid precursor protein by the transmembrane aspartic protease BACE. Science 286, 735-741. doi: 10.1126/science.286.5440.735

Venkatesh, B., Kirkness, E. F., Loh, Y. H., Halpern, A. L., Lee, A. P., Johnson, J., et al. (2007). Survey sequencing and comparative analysis of the elephant shark (Callorhinchus milii) genome. PLoS Biol. 5:e101. doi: 10.1371/journal.pbio.0050101

Venugopal, C., Demos, C. M., Rao, K. S., Pappolla, M. A., and Sambamurti, K. (2008). Beta-secretase: structure, function, and evolution. CNS Neurol. Disord. Drug Targets 7, 278-294. doi: 10.2174/187152708784 936626

Vetrivel, K. S., Meckler, X., Chen, Y., Nguyen, P. D., Seidah, N. G., Vassar, R., et al. (2009). Alzheimer disease Abeta production in the absence of S-palmitoylationdependent targeting of BACE1 to lipid rafts. J. Biol. Chem. 284, 3793-3803. doi: 10.1074/jbc.M808920200

Weiss, M. M., Williamson, T., Babu-Khan, S., Bartberger, M. D., Brown, J., Chen, K., et al. (2012). Design and preparation of a potent series of hydroxyethylamine containing $\beta$-secretase inhibitors that demonstrate robust reduction of central $\beta$-amyloid. J. Med. Chem. 55, 9009-9024. doi: 10.1021/ jm300119p

Xia, W., Wong, S. T., Hanlon, E., and Morin, P. (2012). $\gamma$-Secretase modulator in Alzheimer's disease: shifting the end. J. Alzheimer's Dis. 31, 685-696. doi: 10.3233/Jad-2012-120751
Yan, R., Bienkowski, M. J., Shuck, M. E., Miao, H., Tory, M. C., Pauley, A. M., et al. (1999). Membrane-anchored aspartyl protease with Alzheimer's disease betasecretase activity. Nature 402, 533-537. doi: 10.1038/990107

Zakon, H. H. (2012). Adaptive evolution of voltage-gated sodium channels: the first 800 million years. Proc. Natl. Acad. Sci. U.S.A. 109(Suppl.), 10619-10625. doi: 10.1073/pnas.1201884109

Conflict of Interest Statement: The authors declare that the research was conducted in the absence of any commercial or financial relationships that could be construed as a potential conflict of interest.

Received: 21 September 2013; accepted: 29 November 2013; published online: 17 December 2013.

Citation: Southan C and Hancock JM (2013) A tale of two drug targets: the evolutionary history of BACE1 and BACE2. Front. Genet. 4:293. doi: 10.3389/fgene. 2013.00293

This article was submitted to Bioinformatics and Computational Biology, a section of the journal Frontiers in Genetics.

Copyright (c) 2013 Southan and Hancock. This is an open-access article distributed under the terms of the Creative Commons Attribution License (CC BY). The use, distribution or reproduction in other forums is permitted, provided the original author(s) or licensor are credited and that the original publication in this journal is cited, in accordance with accepted academic practice. No use, distribution or reproduction is permitted which does not comply with these terms. 